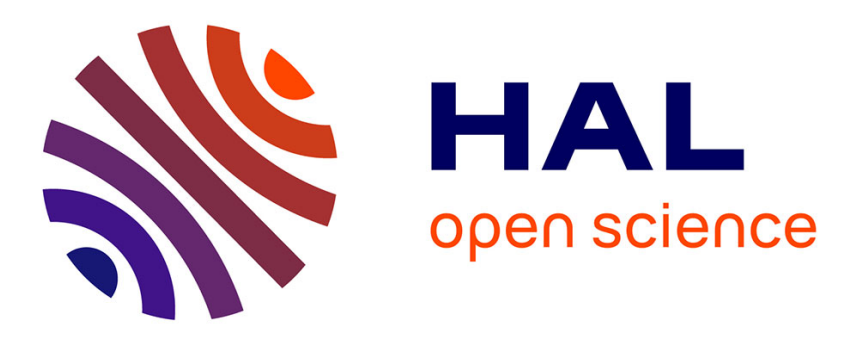

\title{
6D interaction control with aerial robots: The flying end-effector paradigm
}

Markus Ryll, Giuseppe Muscio, Francesco Pierri, Elisabetta Cataldi, Gianluca Antonelli, Fabrizio Caccavale, Davide Bicego, Antonio Franchi

\section{- To cite this version:}

Markus Ryll, Giuseppe Muscio, Francesco Pierri, Elisabetta Cataldi, Gianluca Antonelli, et al.. 6D interaction control with aerial robots: The flying end-effector paradigm. The International Journal of Robotics Research, 2019, 38 (9), pp.1045-1062. 10.1177/0278364919856694 . hal-02383394

\author{
HAL Id: hal-02383394 \\ https://hal.laas.fr/hal-02383394
}

Submitted on 27 Nov 2019

HAL is a multi-disciplinary open access archive for the deposit and dissemination of scientific research documents, whether they are published or not. The documents may come from teaching and research institutions in France or abroad, or from public or private research centers.
L'archive ouverte pluridisciplinaire HAL, est destinée au dépôt et à la diffusion de documents scientifiques de niveau recherche, publiés ou non, émanant des établissements d'enseignement et de recherche français ou étrangers, des laboratoires publics ou privés. 


\title{
6D Interaction Control with Aerial Robots: The Flying End-Effector Paradigm
}

\author{
Markus RylI ${ }^{1}$, Giuseppe Muscio ${ }^{2}$, Francesco Pierri $^{2}$, Elisabetta Cataldi ${ }^{3}$, Gianluca \\ Antonelli $^{3}$, Fabrizio Caccavale ${ }^{2}$, Davide Bicego ${ }^{1}$ and Antonio Franchi ${ }^{1}$
}

\begin{abstract}
This paper presents a novel paradigm for physical interactive tasks in aerial robotics allowing to increase reliability and decrease weight and costs compared to state of the art approaches. By exploiting its tilted propeller actuation, the robot is able to control the full 6D pose (position and orientation independently) and to exert a full-wrench (force and torque independently) with a rigidly attached end-effector. Interaction is achieved by means of an admittance control scheme in which an outer loop control governs the desired admittance behavior (i.e., interaction compliance/stiffness, damping, and mass) and an inner loop based on inverse dynamics ensures full 6D pose tracking. The interaction forces are estimated by a IMU-enhanced momentum based observer. An extensive experimental campaign is performed and four case studies are reported. Firstly, a hard touch and slide on a wooden surface, named sliding surface task. Secondly, a tilted peg-in-hole task, i.e., the insertion of the end-effector in a tilted funnel. Then an admittance shaping experiment in which it is shown how the stiffness, damping and apparent mass can be modulated at will. Finally, the fourth experiment is in charge of showing the effectiveness of the approach also in the presence of time-varying interaction forces
\end{abstract}

\section{Keywords}

Aerial robotics, Aerial physical interaction, Aerial Manipulation

\section{INTRODUCTION}

Direct physical interaction of a robot with its environment is a vast and continuously growing field of research with several relevant applications. Industrial case studies have been object of massive research, see, e.g., Villani and De Schutter (2008) for an introductory reading. Recently, the use of aerial robotic platforms, possibly equipped with an arm, came prominently into focus. Aerial robots offer a nearly unlimited motion space and can be exposed in dangerous or poisonous environments. Along this line, aerial robots have been recently exploited in operations such as, e.g., transportation (Fink et al. (2011)), structure assembly, object grasping (Augugliaro et al. (2014)), and wall inspection (Fumagalli et al. (2012)), requiring both autonomous flight competences and physical interaction capabilities; the latter being a particularly challenging task due to the complexity of aerodynamics - especially when the vehicle is close to surfaces - and intrinsic instability of almost all the aerial robotic platforms.

To perform physical interaction, aerial robots are either equipped with a rigid tool or an $n$ degree of freedom (DoF) robotic arm. In the first case, the tool is rigidly fixed to the airframe, see, e.g., Gioioso et al. (2014b); Augugliaro et al. (2014); Yüksel et al. (2014b); Nguyen and Lee (2013); Gioioso et al. (2014a). A drawback of this solution is that typical aerial platforms are underactuated and therefore it is impossible to independently control the 6D (position plus orientation) dynamics of the end-effector. This limits the potential use cases and also creates stability issues. In fact, it has been shown that in the presence of interaction with points of the airframe different from the vehicle's center of mass $(\mathrm{CoM})$ the internal dynamics of underactuated multi-rotors is not guaranteed to be stable, and it is, in general, neither easy to stabilize nor practical for real applications (Nguyen and Lee (2013)).

The second possibility is to attach an $n$-DoF robotic arm to the aerial platform (Muscio et al. (2016, 2017); Baizid et al. (2016); Kim et al. (2013); Tognon et al. (2017)), a solution which aims at overcoming the underactuation of the end-effector dynamics by exploiting the increased number of actuators provided by the arm. In this way, a fully actuated 6D force control at the end-effector side becomes possible (Yang and Lee (2014)). However, this solution comes with several drawbacks as well, which are mentioned in the following:

i) a robotic arm strongly decreases the payload and flight time due to its own weight;

ii) the system is much more complex from a mechanical point of view than a single airframe with a rigid tool and, thus, it is more expensive to build and also requires more maintenance and repairing costs across its operational life;

\footnotetext{
${ }^{1}$ LAAS-CNRS, Université de Toulouse, CNRS, Toulouse, France,

${ }^{2}$ University of Basilicata, School of Engineering, via dell'Ateneo Lucano, 10, Potenza, Italy,

${ }^{3}$ University of Cassino and Southern Lazio, Via Di Biasio 43, 03043 Cassino, Italy
}

\section{Corresponding author:}

Antonio Franchi, LAAS-CNRS, Université de Toulouse, CNRS, Toulouse, France.

Email: antonio.franchi@laas.fr 
iii) lateral forces in body frame, which cannot be provided by the aerial platform itself, have to be generated through the dynamical/inertial coupling between the arm and the aerial robot: the proper mastering of the dynamical coupling is something that has to be necessarily exploited in order to get the sought benefits in terms of 6D force control. This, in turn, requires the knowledge of the precise dynamical model and a very accurate measurement of the system inputs and states (position, orientation, linear and angular velocities). As a matter of fact, these requirements are extremely hard to achieve in real world conditions (especially the former). For this reason, kinematic-only approaches have been preferred for real world validations, see e.g., Muscio et al. (2016, 2017), at the expense of losing the main benefits for which the manipulator was introduced.

As a byproduct, the use of such complex systems has been exploited in real world so far only for basic interaction tasks such as, e.g., pick and place, vertical insertion, and pull/pushing of constrained objects like drawers (Kim et al. 2015). At the best of our knowledge, more complex interactions required in real world like: $a$ ) peg in-hole with non-vertical orientation, $b$ ) position-force control for sliding on surfaces, and, more in general, $c$ ) an accurate shaping of the full $6 \mathrm{D}$ force/moment interaction of the endeffector with the environment; have not yet been successfully demonstrated in real world in state-of-the-art systems.

Summarizing, standard flying platforms are underactuated and, thus, incapable of 6D end-effector force control. On the other side, using a full manipulator up in the air to perform the sought $6 \mathrm{D}$ end-effector force control is often excessively complex and may introduce more problems than benefits.

To solve all these problems at once and finally achieve the sought full 6D force control of the aerial interaction, we propose a novel paradigm which we named the Flying End-Effector. The concept is based on the ascertainment that the overall mission goal is to achieve 6D interaction with an end-effector. While in ground robotics the endeffector must be actuated by a manipulator with at least six degrees of freedom $(\mathrm{DoF})$, in the aerial robotics case there is no necessity to bring up to the air a full manipulator together with the end-effector. What is instead needed is that the aerial robot possesses the minimal requirements to perform such interaction with a rigidly attached endeffector. In order to achieve such requirements, we propose to use a multi-rotor robot with non-collinear fixedly-tilted propellers (NCFTP) instead of the more common collinear fixedly-tilted propeller (CFTP) architectures (Ryll et al. (2016)). In NCFTP platforms, which appeared in the robotics literature only recently (see, e.g., Rajappa et al. (2015); Voyles and Jiang (2014); Brescianini and D'Andrea (2016); Park et al. (2016)), the full-actuation is achieved by a more general propeller position and orientation. The difference between the underactuated platform and NCFTP platforms is that, in the former approach, all the propellers have the same orientation while, in the latter approach, every propeller orientation is different. The NCFTP approach is, thus, able to control independently the translational and angular acceleration when unconstrained, or any of the six components of the exerted wrench when in contact, thus allowing full and dexterous 6D force control, which makes them much more suited for physical interaction tasks than standard CFTP platforms.

Another solution to obtain full-actuation consists of actively tilting the whole propeller groups (Ryll et al. (2016, 2015); Long and Cappelleri (2013)), a solution which is called thrust vectoring or tilting propeller. This solution however is subject to the same drawbacks of the solutions employing a manipulator arm, since they require extra actuation, mechanical complexity, and weight. Furthermore, they cannot in general guarantee instantaneous disturbance rejection or fast force exertion since the propellers might have to be re-oriented, which again takes some nonnegligible time.

A critical issue for aerial robot interaction control is the measurement of the interaction wrench. A reliable solution is the adoption of force/torque sensors, such as in Antonelli et al. (2016) where the wrench measurement of a wrist mounted sensor of an aerial manipulator is fed to an admittance filter. Use of force/torque sensor increases the cost and the weight of the aerial platform, thus alternative solutions based on wrench estimators have been proposed in the last years. In Yüksel et al. (2014a) a Lyapunovbased nonlinear observer is proposed for estimating the external wrenches applied on a quadrotor, while in Tomic and Haddadin (2014) a hybrid estimation is proposed, using the linear acceleration for directly computing the interaction forces and a momentum based observer for estimating the interaction torques. In Tomic et al. (2017) the same authors propose a more refined hybrid estimation, where the estimated forces are not simply computed by the model but are obtained via a first-order stable filter, similar to the solution proposed in Yüksel et al. (2014a). In Rajappa et al. (2017), the authors, by exploiting both a wrench estimation and a ring of eight contact sensors, proposed a control able to separate human interaction forces from additional disturbances as wind and parameter uncertainties. In Tagliabue et al. (2016) as well an admittance control framework is used for collaborative transportation of objects by using underactuated aerial robots, an approach which is theoretically analyzed and refined in Tognon et al. (2018) by demonstrating its stability and the fundamental role of the internal force in such control scheme.

A preliminary (6-pages long) version of this work has been presented in a conference version (Ryll et al. (2017)). With respect to Ryll et al. (2017), in this paper $i$ ) we provide much more details for all considered aspect; ii) we consider an improved solution for the wrench estimate that exploits also the linear acceleration measurements provided by the IMU; iii) the position and force tracking quality has been largely improved; $i v$ ) the physical property shaping capabilities of the admittance filter are thoroughly tested and explained and v) a very broad range of experiments is performed and discussed; in particular, the case of sliding on a surface with a tilted orientation, the peg-in-hole experiment, and the admittance shaping tests and sliding in contact with two ledges have been conducted.

Concluding this introduction, in the following we summarize the main contributions (but not all) of this work: 1) we propose and show the practicability through real experiments, of a completely new aerial physical interaction paradigm: the $6 \mathrm{D}$ flying end-effector. We believe that this 


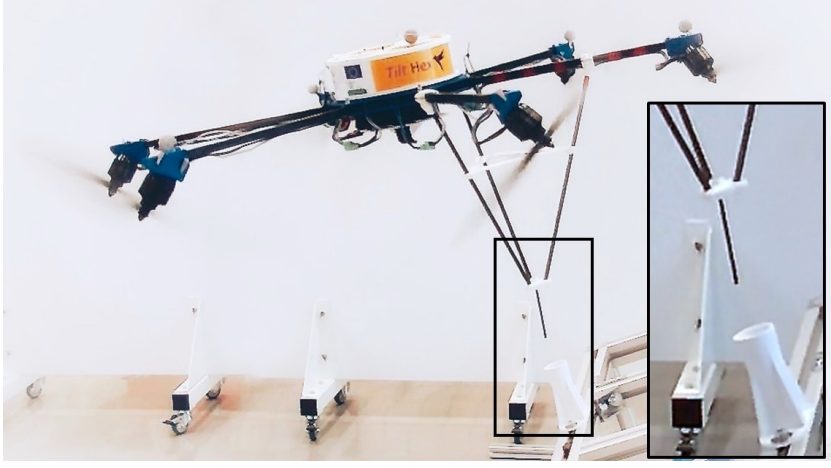

Figure 1. The Tilt-Hex (NCFTP aerial platform with tilted propellers in-house developed at LAAS-CNRS) with the rigidly attached end-effector. Notice how the interaction takes place far away and off-centered from the vehicle CoM. The picture is taken right after a Peg-In-Hole task was successfully performed.

paradigm will pave the way to a novel aerial system concept which outperforms currently adopted solutions for aerial manipulation and physical interaction in terms of capability, reliability, complexity and costs.

2) we propose a specific integration of known robotic algorithms, achieving both motion and interaction control as well as external wrench estimation, in a thoroughly conceived architecture and show how the integrated system can work with a minimal sensor suite (pose sensor plus IMU) not even needing a force sensor in its basic configuration - the addition of more sensors, if available, being anyway straightforward.

3 ) in support of the aforementioned features, we show experiments that are the first of their kind in aerial robotics: fully impedance shaping in $6 \mathrm{D}$, peg-in-hole with tilted holes, sliding on tilted surfaces. Moreover, in order to show the effectiveness of the wrench estimate, a forth experiment, consisting on sliding in contact with two ledges on a surface mounted on an ATI45 force/torque sensor, has been conducted in such a way to exert variable forces and torques on the end-effector. The wrench estimator results are compared with the measures obtained by the sensor. The experiments are designed to clearly show the versatility and the robustness of the proposed approach to the environmental uncertainties.

The paper is organized as follows. First a generic model for NCFTP aerial systems is introduced and afterwards we model the proposed NCFTP platform, named Tilt-Hex. Then the complete admittance control framework is presented as well as its components, namely the inner loop geometric pose controller, the wrench observer and the outer loop admittance filter. Then we present the hard-/software architecture and the experimental evaluation. Finally, we conclude the paper with a summary of the results and an outline of future works.

Remark It is important to mention that the theory and the architecture proposed in this paper is very general and works seamlessly with any NCFTP platform other than the one used in particular here.

\section{System Modeling}

We consider as aerial robot a fully actuated aerial vehicle equipped with an arbitrarily mounted end-effector tool. The presented physical interaction framework is generic for any fully actuated system able to track a full-pose trajectory with the end-effector. We shall start with the generic parts of the modeling and present afterwards the instantiation of this general model for the NCFTP hexarotor used in the experiments.

General Modeling Let us consider the following coordinate frames (see Figure 2):

- Inertial world frame $\mathscr{F}_{W}$, whose axes (unit vectors) and origin are indicated with $\left\{\mathbf{x}_{W}, \mathbf{y}_{W}, \mathbf{z}_{W}\right\}$ and $O_{W}$, respectively;

- Body frame $\mathscr{F}_{R}: O_{R}-\left\{\mathbf{x}_{R}, \mathbf{y}_{R}, \mathbf{z}_{R}\right\}$, attached to the robot and with origin $O_{R}$ in the CoM of the aerial vehicle with the end-effector;

- End-effector frame $\mathscr{F}_{E}: O_{E}-\left\{\mathbf{x}_{E}, \mathbf{y}_{E}, \mathbf{z}_{E}\right\}$, attached to the robot end effector and with origin in the interaction point $O_{E}$.

For a generic vector $v$, the notation $v^{\star}$ (with $\star=R, E$ ) denotes that the vector $v$ is expressed in frame $\mathscr{F}_{\star}$. If the superscript is omitted it means that the vector is expressed in inertial frame.

The position of $O_{R}$ expressed in $\mathscr{F}_{W}$ is denoted by $\mathbf{p}_{R} \in$ $\mathbb{R}^{3}$, while the position of $O_{E}$ in $\mathscr{F}_{W}$ and in $\mathscr{F}_{R}$ are denoted by $\mathbf{p}_{E} \in \mathbb{R}^{3}$ and $\mathbf{p}_{E}^{R} \in \mathbb{R}^{3}$, respectively, where $\mathbf{p}_{E}^{R}$ is constant over time. Analogously, let us denote with $\mathbf{R}_{R} \in S O(3)$ and $\mathbf{R}_{E} \in$ $S O(3)\left(\right.$ where $\left.S O(3)=\left\{\mathbf{A} \in \mathbb{R}^{3 \times 3} \mid \mathbf{A} \mathbf{A}^{\mathrm{T}}=\mathbf{I} \wedge \operatorname{det} \mathbf{A}=1\right\}\right)$ the rotation matrices expressing, respectively, the orientation of frame $\mathscr{F}_{R}$ and $\mathscr{F}_{E}$ with respect to the inertial frame $\mathscr{F}_{W}$. Moreover, $\mathbf{R}_{E}^{R} \in S O(3)$ denotes the constant rotation matrix expressing the orientation of $\mathscr{F}_{E}$ with respect to $\mathscr{F}_{R}$. Finally let us denote as $\boldsymbol{\omega}_{R}^{R} \in \mathbb{R}^{3}$ and $\boldsymbol{\omega}_{E}^{E} \in \mathbb{R}^{3}$ the angular velocities of $\mathscr{F}_{R}$ and $\mathscr{F}_{E}$ with respect to $\mathscr{F}_{W}$ expressed, respectively, in frame $\mathscr{F}_{R}$ and $\mathscr{F}_{E}$. Thus, the orientation kinematics of the robot and the end effector are then expressed by

$$
\begin{aligned}
\dot{\mathbf{R}}_{R} & =\mathbf{R}_{R}\left[\boldsymbol{\omega}_{R}^{R}\right]_{\times} \\
\dot{\mathbf{R}}_{E} & =\mathbf{R}_{E}\left[\boldsymbol{\omega}_{E}^{E}\right]_{\times},
\end{aligned}
$$

respectively, where $[\bullet]_{\times} \in S O(3)$ represents the skew symmetric matrix (Siciliano et al. (2009)) associated to vector $\bullet \in \mathbb{R}^{3}$.

Using the Newton-Euler formalism, the equation of motion of the aerial robot can be expressed as

$$
\left[\begin{array}{c}
m \ddot{\mathbf{p}}_{R} \\
\mathbf{J} \dot{\boldsymbol{\omega}}_{R}^{R}
\end{array}\right]=\underbrace{-\left[\begin{array}{c}
m g \mathbf{e}_{3} \\
\boldsymbol{\omega}_{R}^{R} \times \mathbf{J} \boldsymbol{\omega}_{R}^{R}
\end{array}\right]}_{\mathbf{g}\left(\boldsymbol{\omega}_{R}^{R}\right)}+\left[\begin{array}{c}
\mathbf{f} \\
\boldsymbol{\tau}^{R}
\end{array}\right]+\left[\begin{array}{c}
\mathbf{f}_{R} \\
\boldsymbol{\tau}_{R}^{R}
\end{array}\right]
$$

where $m$ and $\mathbf{J} \in \mathbb{R}^{3 \times 3}$ represent the robot mass and its inertia matrix with respect to $O_{R}$ and expressed in $\mathscr{F}_{R}$, respectively, $g$ is the gravitational acceleration, $\mathbf{f} \in \mathbb{R}^{3}$ and $\tau^{R} \in \mathbb{R}^{3}$ are force and torque inputs, while $\mathbf{f}_{R}$ and $\tau_{R}^{R}$ are the external force and torque on the robot CoM due to the force and torque exerted by the environment on the end-effector. 


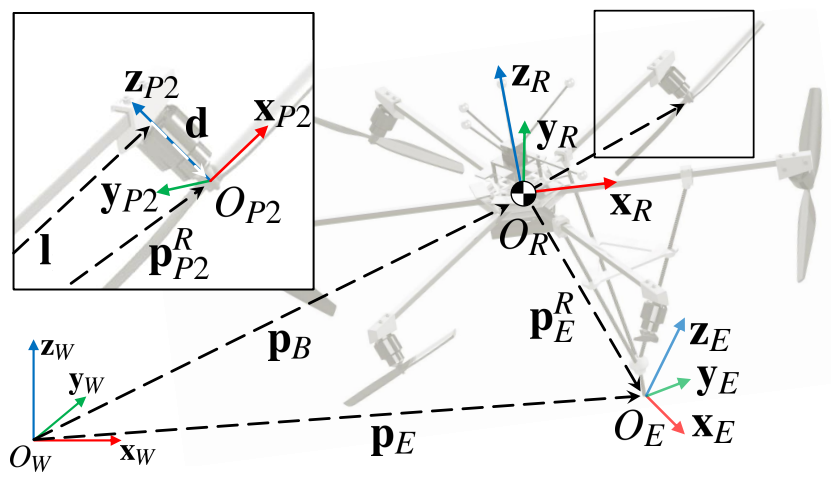

Figure 2. Schematic view of important frames and vectors of the Tilt-Hex with the rigidly attached end-effector. The zoomed propeller group shows further vectors needed to model the system.

Remark It is worth noticing that, as usual in aerial robotics field, in (3) the translational dynamics is expressed in the frame $\mathscr{F}_{W}$, while the rotational dynamics is expressed in the frame $\mathscr{F}_{R}$.

Tilt-Hex Without loss of generality for any fully actuated aerial platform, we will now derive (3) for the NCFTP platform used in the later presented experiments. The NCFTP platform is based on a hexarotor structure, with propellers equally-spaced and equidistant from $O_{R}$ in the $\mathrm{x}$-y-plane of $\mathscr{F}_{R}$. Full actuation is achieved by rigid adapters, tilting every single motor-propeller combination (see Figure 1 and 2). Let us consider 6 frames $\mathscr{F}_{P i}, i=1 \ldots 6$ where $O_{P_{i}}$ coincides with the center of rotation of the $i$-th propeller group (see Figure 2). The orientation of $\mathscr{F}_{P i}$ with respect to $\mathscr{F}_{B}$ is represented by the rotation matrix

$$
\mathbf{R}_{P_{i}}^{R}=\mathbf{R}_{z}\left((i-1) \frac{\pi}{3}\right) \mathbf{R}_{x}\left((-1)^{i-1} \alpha\right) \mathbf{R}_{y}(\beta), \quad i=1 \ldots 6 .
$$

The inclination of the $i$-th motor-propeller group with respect to $\mathscr{F}_{R}$ is defined by the constant parameters $\alpha$ and $\beta$. The selection of $\alpha$ and $\beta$ decides the maximum lateral forces with the cost that higher lateral forces result in higher internal forces and therefore a waste of energy (Rajappa et al. (2015)). Selecting an alternating sign of $\alpha$ for every other propeller in (4) allows for the full actuation of the aerial vehicle.

The $i$-th motor-propeller group's position with respect to $O_{R}$ can be defined as

$$
\mathbf{p}_{P_{i}}^{R}=\mathbf{R}_{z}\left((i-1) \frac{\pi}{3}\right) \mathbf{l}+\mathbf{R}_{x}\left((-1)^{i-1} \alpha\right) \mathbf{R}_{y}(\beta) \mathbf{d}, \quad i=1 \ldots 6
$$

where $\mathbf{d}$ is the vector from the center of the tilting rotation to the center of the motor-propeller group $O_{P_{i}}$, and $\mathbf{l}$ is the vector from $O_{R}$ to the center of the tilting rotation (see Figure 2).

As well known, a spinning propeller generates a thrust force and a drag moment in $O_{P_{i}}$. In a good approximation both can be modeled by utilizing the signed squared of the spinning velocity as

$$
\mathbf{f}_{i}^{R}=c_{f} u_{i} \mathbf{R}_{P_{i}}^{R} \mathbf{e}_{3}, \quad i=1 \ldots 6
$$

and

$$
\tau_{i}^{R}=(-1)^{i-1} c_{\tau} u_{i} \mathbf{R}_{P_{i}}^{R} \mathbf{e}_{3}, \quad i=1 \ldots 6
$$

\begin{tabular}{|c|c|c|}
\hline Definition & Symbol & Value \\
\hline Inertial world frame with origin $O_{W}$ and axes $\left\{\mathbf{x}_{W}, \mathbf{y}_{W}, \mathbf{z}_{W}\right\}$ & $\mathscr{F}_{W}$ & \\
\hline Robot body frame with origin $O_{R}$ and axes $\left\{\mathbf{x}_{R}, \mathbf{y}_{R}, \mathbf{z}_{R}\right\}$ & $\mathscr{F}_{R}$ & \\
\hline End effector frame with origin $O_{E}$ and axes $\left\{\mathbf{x}_{E}, \mathbf{y}_{E}, \mathbf{z}_{E}\right\}$ & $\mathscr{F} E$ & \\
\hline Symbols that can assume the values $W, R$, or $E$ & $\star, \circ$ & \\
\hline Position of $O_{\circ}$ in $\mathscr{F}_{W}$ & $\mathbf{p}_{\circ}$ & \\
\hline Velocity of $O_{\circ}$ in $\mathscr{F}_{W}$ & $\mathbf{v}_{\circ}$ & \\
\hline Rotation matrix expressing the orientation of $\mathscr{F}_{\circ}$ w.r.t. $\mathscr{F}_{\star}$ & $\mathbf{R}_{\circ}^{\star}$ & \\
\hline Rotation matrix expressing the orientation of $\mathscr{F}_{\circ}$ w.r.t. $\mathscr{F}_{W}$ & $\mathbf{R}_{\circ}$ & \\
\hline Angular velocity of $\mathscr{F}_{\circ}$ w.r.t. $\mathscr{F}_{W}$, expressed in $\mathscr{F}_{\circ}$ & $\omega_{\circ}$ & \\
\hline Reference position expressed in $\mathscr{F}_{W}$ & $\mathbf{p}_{\mathrm{R}, \mathrm{r}}$ & \\
\hline Reference rotation matrix & $\mathbf{R}_{\mathrm{R}, \mathrm{r}}$ & \\
\hline Desired position expressed in $\mathscr{F}_{W}$ & $\mathbf{p}_{\mathrm{R}, \mathrm{d}}$ & \\
\hline Desired rotation matrix & $\mathbf{R}_{\mathrm{R}, \mathrm{d}}$ & \\
\hline Actuation wrench expressed in $\mathscr{F}_{W}$ & $\mathbf{w}$ & \\
\hline Real external wrench on the EE in $\mathscr{F}_{W}$ & $\mathbf{w}_{E}$ & \\
\hline Observed (estimated) external wrench on the EE in $\mathscr{F}_{W}$ & $\hat{\mathbf{w}}_{E}$ & \\
\hline Observed (estimated) external wrench on the robot in $\mathscr{F}_{W}$ & $\hat{\mathbf{w}}_{R}$ & \\
\hline Tilting angle (around $\mathbf{x}_{P_{i}}$ ) of the $i^{t h}$ prop. group & $\alpha_{i}$ & $35^{\circ}$ \\
\hline Tilting angle (around $\mathbf{y}_{P_{i}}$ ) of the $i^{\text {th }}$ prop. group & $\beta_{i}$ & $10^{\circ}$ \\
\hline$i^{\text {th }}$ propeller blade spinning frequency about $\mathbf{z}_{P_{i}}$ (in $\mathrm{Hz}$ ) & $\sqrt{u}_{i}$ & \\
\hline Mass of the whole aerial robot & $m$ & $2.4 \mathrm{Kg}$ \\
\hline Gravity acceleration constant & $g$ & $9.81 \mathrm{~m} / \mathrm{s}^{2}$ \\
\hline Gain matrix of the wrench observer & $\mathbf{K}_{I}$ & \\
\hline Mechanical impedance inertia matrix & $\mathbf{M}_{E}$ & \\
\hline Mechanical impedance damping matrix & $\mathbf{D}_{E}$ & \\
\hline Mechanical impedance stiffness matrix & $\mathbf{K}_{E}$ & \\
\hline
\end{tabular}

Table 1. Overview of most symbols used in this paper. If constant through all experiments a value is presented as well.

with $c_{f}$ and $c_{\tau}$ being constant parameters linking the propeller spinning velocity $\sqrt{u_{i}}$ to the generated thrust force and drag moment. The term $(-1)^{i-1}$ in (7) represents the effect of counter spinning rotors for all even propellers.

We can now express the total force applied on $O_{R}$ in $\mathscr{F}_{W}$ as

$$
\mathbf{f}(\mathbf{u})=\mathbf{R}_{R} \sum_{i=1}^{6} \mathbf{f}_{i}^{R}=\mathbf{R}_{R} \mathbf{F}_{1} \mathbf{u}
$$

with $\mathbf{F}_{1} \in \mathbb{R}^{3 \times 6}$ incorporating the geometrical and physical properties of the aerial robot (i.e., dimensions, tilting angles, thrust coefficients) and with $\mathbf{u}=\left[u_{1} \ldots u_{6}\right]^{\mathrm{T}}$ being the vector collecting the 6 squared propeller spinning velocities.

In the same manner we can incorporate the total torque due to the thrust contribution and the drag moment expressed in $\mathscr{F}_{B}$ utilizing (6) and (7) as

$$
\boldsymbol{\tau}^{R}(\mathbf{u})=\sum_{i=1}^{6}\left(\mathbf{p}_{P}^{R} \times \mathbf{f}_{i}^{R}+\boldsymbol{\tau}_{i}^{R}\right)=\mathbf{F}_{2} \mathbf{u}
$$

where $\mathbf{F}_{2} \in \mathbb{R}^{3 \times 6}$ again includes the geometrical and physical properties. A detailed derivation of the model and of $\mathbf{F}_{1}$ and $\mathbf{F}_{2}$ and its necessary conditions for full actuation can be found in Rajappa et al. (2015) and Michieletto et al. (2017). By replacing (8) and (9) in (3) we obtain

$$
\left[\begin{array}{c}
m \ddot{\mathbf{p}}_{R} \\
\mathbf{J} \dot{\boldsymbol{\omega}}_{R}^{R}
\end{array}\right]=\mathbf{g}\left(\boldsymbol{\omega}_{R}\right)+\left[\begin{array}{c}
\mathbf{R}_{R} \mathbf{F}_{\mathbf{1}} \\
\mathbf{F}_{\mathbf{2}}
\end{array}\right] \mathbf{u}+\left[\begin{array}{c}
\mathbf{f}_{R} \\
\tau_{R}^{R}
\end{array}\right]
$$

as a reliable dynamical model under slow flight conditions $(<0.5 \mathrm{~m} / \mathrm{s})$. We neglect any aerodynamic effects like the well known first order-effects rotor drag, fuselage drag and H-force as these effects linearly depend on the vehicle's velocity and can therefore be neglected for the slow velocity aerial interaction considered in this paper (Faessler et al. (2018); Kai et al. (2017)). 


\section{Controller}

In this section we describe the single components of the controller. The control framework is based on an outer loop admittance control and an inner loop full-pose controller (see Figure 5). The state of the aerial robot is estimated by a Unscented Kalman Filter (UKF) that fuses the Inertial Measurement Unit (IMU) acceleration and angular velocity measurements with the position and orientation from a pose sensor (in our case a motion capture system, which could be easily replaced with an onboard camera using a Perspectiven-Point (PnP) algorithm). The interaction torques and forces are estimated by a wrench observer.

\section{Pose Controller}

The inner loop, the pose-control law aims at finding the six desired propeller spinning velocities $\mathbf{u} \in \mathbb{R}^{6}$ that will let $\mathbf{p}_{R}$ and $\mathbf{R}_{R}$ track an arbitrary full-pose reference trajectory $\left(\boldsymbol{p}_{R, r}(t), \boldsymbol{R}_{R, r}(t)\right): \mathbb{R} \rightarrow \mathbb{R}^{3} \times S O(3)$. A further application of the full pose controller is presented in Rajappa et al. (2015), where it was first presented. We will therefore only summarize the controller here.

By neglecting external forces and torques acting on the aerial robot's structure or end-effector the dynamical model in can be rewritten as

$$
\left[\begin{array}{c}
\ddot{\mathbf{p}}_{R} \\
\dot{\boldsymbol{\omega}}_{R}^{R}
\end{array}\right]=\mathbf{g}\left(\boldsymbol{\omega}_{R}\right)+\mathbf{J}_{R} \mathbf{u},
$$

where $\boldsymbol{g}\left(\omega_{R}\right) \in \mathbb{R}^{6}$ is a drift vector, combing the gravitational and Coriolis effect and $\mathbf{J}_{R}=\left[\begin{array}{cc}\frac{1}{m} \mathbf{R}_{R} \mathbf{F}_{1} & \mathbf{0} \\ \mathbf{0} & \mathbf{J}^{-1} \mathbf{F}_{2}\end{array}\right] \in \mathbb{R}^{6 \times 6}$. We will refer to $\mathbf{J}_{R}$ as the robot's input Jacobian of the inner loop system*.

If the input Jacobian $\boldsymbol{J}_{R}$ is invertible, we can select

$$
\mathbf{u}=\mathbf{J}_{R}^{-1}(\alpha, \beta)\left(-\mathbf{g}\left(\omega_{R}\right)+\mathbf{v}\right)
$$

as control input with $\mathbf{v}$ as an fictitious, additional input (Rajappa et al. (2015), Franchi et al. (2018)), thus obtaining

$$
\left[\begin{array}{c}
\ddot{\mathbf{p}}_{R} \\
\dot{\boldsymbol{\omega}}_{R}^{R}
\end{array}\right]=\mathbf{v}=\left[\begin{array}{c}
\mathbf{v}_{\ddot{\mathbf{p}}_{R}} \\
\mathbf{v}_{\dot{\boldsymbol{\omega}}_{R}^{R}}^{R}
\end{array}\right],
$$

a statically feedback-linearized system. The block diagram of Figure 3 shows the control scheme architecture.

Aiming for an exponential convergence of the position error $\mathbf{p}_{R, r}-\mathbf{p}_{R}=\mathbf{e}_{p}$ to $\mathbf{0}$ a natural choice is the linear controller

$$
\mathbf{v}_{\ddot{\mathbf{p}}_{R}}=\ddot{\mathbf{p}}_{R, r}+\mathbf{K}_{p_{1}} \dot{\mathbf{e}}_{p}+\mathbf{K}_{p_{2}} \mathbf{e}_{p},
$$

where the diagonal positive-definite gain matrixes $\mathbf{K}_{p_{1}}, \mathbf{K}_{p_{2}}$ define Hurwitz polynomials.

Let's now consider the orientation tracking. A common choice to represent orientation tracking uses Euler angles. However, Euler angles are prone to singularity issues. We therefore directly develop the orientation controller on $S O(3)$ and hereby avoid the common issues of local coordinate frames.

Following (Franchi et al. 2018) (Lee et al. 2010), we assume that $\mathbf{R}_{R, r}(t) \in \overline{\mathscr{C}}^{3}$ and $\boldsymbol{\omega}_{R, r}=\left[\mathbf{R}_{R, r}^{T} \dot{\mathbf{R}}_{R, r}\right]_{\vee}$, where $[\cdot]_{\vee}$ represents the inverse map from $S O(3)$ to $\mathbb{R}^{3}$. The attitude tracking error $\mathbf{e}_{R} \in \mathbb{R}^{3}$ is given by

$$
\mathbf{e}_{R}=\frac{1}{2}\left[\mathbf{R}_{R, r}^{T} \mathbf{R}_{R}-\mathbf{R}_{R}^{T} \mathbf{R}_{R, r}\right] \vee,
$$

and the tracking error of the angular velocity $\mathbf{e}_{\omega} \in \mathbb{R}^{3}$ is given by

$$
\mathbf{e}_{\omega}=\boldsymbol{\omega}_{R}-\mathbf{R}_{R}^{T} \mathbf{R}_{R, r} \boldsymbol{\omega}_{R, r} .
$$

To as well achieve an asymptotic convergence to $\mathbf{0}$ of the rotational error $\mathbf{e}_{R}$ we select the following orientation controller

$$
\mathbf{v}_{\dot{\boldsymbol{\omega}}_{R}^{R}}=\dot{\boldsymbol{\omega}}_{R, r}-\mathbf{K}_{R_{1}} \mathbf{e}_{\omega}-\mathbf{K}_{R_{2}} \mathbf{e}_{R}
$$

where the diagonal positive definite gain matrixes $\mathbf{K}_{R_{1}}, \mathbf{K}_{R_{2}}$ again define Hurwitz polynomials.

\section{Contact Wrench Estimation}

In order to properly handle physical interaction of the aerial robot with the external environment, the knowledge of the contact interaction wrench between the tool tip and the environment, $\mathbf{w}_{E}=\left[\mathbf{f}_{E}^{\mathrm{T}} \boldsymbol{\tau}_{E}^{\mathrm{T}}\right]^{\mathrm{T}} \in \mathbb{R}^{6}$ is essential. To this aim, a force/torque sensor could be mounted on the robot's tooltip, which is usually capable to provide a reliable measure, but this solution increases both the cost and the weight of the robot. In the aerial robotics field, a more viable solution is the adoption of a wrench estimator, that can provide a sufficiently accurate estimation, denoted as $\hat{\mathbf{w}}_{E}=\left[\hat{\mathbf{f}}_{E}^{\mathrm{T}} \hat{\boldsymbol{\tau}}_{E}^{\mathrm{T}}\right]^{\mathrm{T}} \in$ $\mathbb{R}^{6}$, in the presence of accurate measurements of position, velocities and, if available, accelerations.

The external wrench on the robot, $\mathbf{w}_{R}=\left[\mathbf{f}_{R}^{\mathrm{T}}, \mathbf{R}_{R} \tau_{R}^{R \mathrm{~T}}\right]^{\mathrm{T}}$, can be viewed as the effect on the robot CoM of the wrench $\mathbf{w}_{E}$ exerted by the environment on the tool tip, namely

$$
\mathbf{w}_{R}=\mathbf{H}_{E}^{\mathrm{T}}\left(\mathbf{R}_{R}\right) \mathbf{w}_{E}, \quad \mathbf{H}_{E}\left(\mathbf{R}_{R}\right)=\left[\begin{array}{cc}
\mathbf{I}_{3} & -\left[\mathbf{R}_{R} \mathbf{p}_{E}^{R}\right]_{\times} \\
\mathbf{O}_{3} & \mathbf{I}_{3}
\end{array}\right] .
$$

The sensor equipment of the NCFTP platform used in the experiments provides accurate enough measurements of the platform position and velocities, both angular and linear, while only the linear acceleration, provided by the IMU, can be reasonably used in a wrench observer. Thus, in this paper, the hybrid approach already proposed in Tomic et al. (2017), has been followed. More in detail, the acceleration based observer proposed by Yüksel et al. (2014a) is adopted in order to estimate the external interaction forces on the robot $\mathrm{CoM}, \mathbf{f}_{R}$, while the external torques, $\tau_{R}^{R}$ are obtained by exploiting a momentum-based observer (De Luca and Mattone (2005)).

Estimation of contact forces The following disturbance observer requiring the vehicle acceleration measure, firstly proposed for aerial robots in Yüksel et al. (2014a), is adopted for estimating the contact forces

$$
\begin{aligned}
\dot{\hat{\mathbf{f}}}_{R} & =\mathbf{L}\left(\mathbf{f}_{R}-\hat{\mathbf{f}}_{R}\right) \\
& =-\mathbf{L} \hat{\mathbf{f}}_{R}-\mathbf{L}\left(m \ddot{\mathbf{p}}_{R}+m g \mathbf{e}_{3}-\mathbf{R}_{R} \mathbf{F}_{1} \mathbf{u}\right),
\end{aligned}
$$

where $\mathbf{L} \in \mathbb{R}^{3 \times 3}$ is a gain matrix to be designed and $\hat{\mathbf{f}}_{R}$ is an estimate of $\mathbf{f}_{R}$. By defining the observer error as $\mathbf{e}_{f}=\mathbf{f}_{R}-\hat{\mathbf{f}}_{R}$,

*In standard hexarotor the input Jacobian $\boldsymbol{J}_{R}(\alpha, \beta)$ has rank equal to four, similar to a quadrotor. 


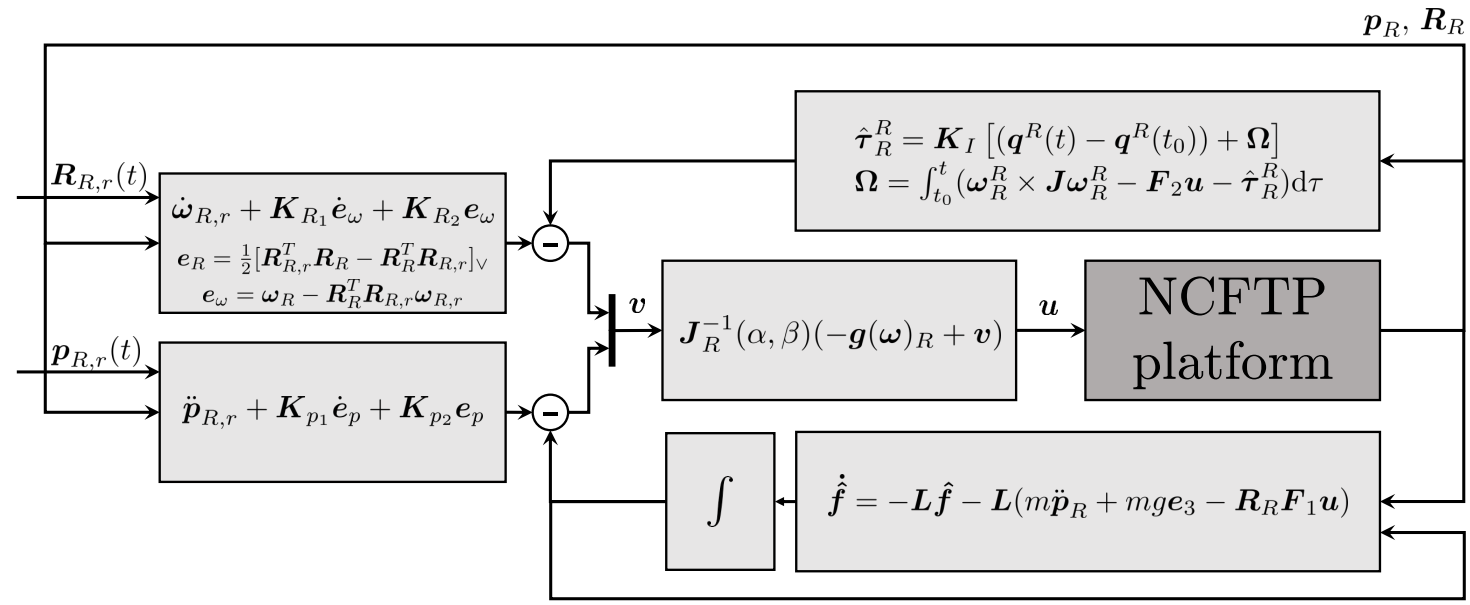

Figure 3. Block diagram of the inner loop, low level pose controller.

the error dynamics, in the presence of a constant or slowly varying external force is given by Yüksel et al. (2014a)

$$
\dot{\mathbf{e}}_{f}+\mathbf{L} \mathbf{e}_{f}=\mathbf{0}_{3}
$$

where $\mathbf{0}_{3} \in \mathbb{R}^{3}$ is the $(3 \times 1)$ null vector. Thus, the error dynamics is exponentially convergent to the origin for any positive definite matrix $\mathbf{L}$.

Contact torques estimation In order to estimate the interaction torques, exerted by the external environment on the tool-tip, a momentum-based observer (De Luca and Mattone (2005)) has been designed. With reference to the system dynamics (10), the angular momentum $\mathbf{q}^{R} \in \mathbb{R}^{3}$ in frame $\mathscr{F}_{R}$ can be computed as

$$
\mathbf{q}^{R}=\mathbf{J} \boldsymbol{\omega}_{R}^{R}
$$

From (3), the time-derivative of (21) can be expressed as

$$
\dot{\mathbf{q}}^{R}=\mathbf{J} \dot{\boldsymbol{\omega}}_{R}^{R}=-\boldsymbol{\omega}_{R}^{R} \times \mathbf{J} \boldsymbol{\omega}_{R}^{R}+\mathbf{F}_{2} \mathbf{u}+\boldsymbol{\tau}_{R}^{R}
$$

By exploiting (22), the estimate $\hat{\tau}_{R}$ can be seen as the residual vector

$$
\hat{\boldsymbol{\tau}}_{R}^{R}=\mathbf{K}_{I}\left[\left(\mathbf{q}^{R}(t)-\mathbf{q}^{R}\left(t_{0}\right)\right)+\int_{t_{0}}^{t}\left(\boldsymbol{\omega}_{R}^{R} \times \mathbf{J} \boldsymbol{\omega}_{R}^{R}-\mathbf{F}_{2} \mathbf{u}-\hat{\boldsymbol{\tau}}_{R}^{R}\right) \mathrm{d} \tau\right],
$$

where $t$ and $t_{0}$ are the current and initial time instant respectively, $\mathbf{K}_{I}$ is a positive definite gain matrix. By reasonably assuming that $\boldsymbol{\omega}_{R}^{R}\left(t_{0}\right)=\mathbf{0}_{3}$, it implies that $\mathbf{q}^{R}\left(t_{0}\right)$ is null as well. By taking the time derivative of (23), through (22), the following dynamics for the residual vector is obtained

$$
\dot{\hat{\tau}}_{R}^{R}+\mathbf{K}_{I} \hat{\tau}_{R}^{R}=\mathbf{K}_{I} \tau_{R}^{R}
$$

Equation (24) is a first order low-pass dynamic system: it can be easily recognized that $\hat{\tau}_{R}^{R} \rightarrow \tau_{R}^{R}$ when $t \rightarrow \infty$ for any positive definite gain matrix $\mathbf{K}_{I}$. The choice of the matrix $\mathbf{K}_{I}$ is a trade-off between the convergence rate and the filtering properties of the observer: greater values of the gains allow faster convergence while smaller values allow to filter the high-frequency noise.
Wrench acting on the tool tip Once both $\hat{\mathbf{f}}_{R}$ and $\hat{\boldsymbol{\tau}}_{R}^{R}$ are known, the estimated wrench acting on the tool tip, $\hat{\mathbf{w}}_{E}$ is computed as

$$
\hat{\mathbf{w}}_{E}=\mathbf{H}_{E}^{-\mathrm{T}}\left[\begin{array}{c}
\hat{\mathbf{f}}_{R} \\
\mathbf{R}_{R} \hat{\boldsymbol{\tau}}_{R}^{R}
\end{array}\right] .
$$

An illustrative example of the wrench observer's precision is presented in Figure 4. For this test the aerial robot has been rigidly connected to an ATI45 force-torque sensor, which itself has been mounted on a test-stand. By letting the aerial robot tracking a trajectory and only utilizing the inner loop pose controller, the aerial robot applies a force-torque profile defined by the trajectory on the force-torque sensor. To properly test the limits of the wrench observer a chirp signal (cosinus with increasing frequency), simultaneously about multiple axes, has been used as trajectory. The resulting force and torque profiles are presented in Figure 4. For the sake of clarity, the Figure 4-a reports the components of $\hat{\mathbf{f}}_{E}$ and the Figure 4-c presents the components of $\hat{\boldsymbol{\tau}}_{E}$ in (25) (continuous lines) against data of an ATI45 force-torque sensor (dashed lines). It is obvious that the observer can track well multiple signals, while the tracking quality slowly decreases for increasing frequencies (see Figure 4-b and d).

\section{Interaction wrench compensation}

To achieve optimal results of the admittance filter a highly stiff low-level tracking is desired. This could be achieved by increasing the gains in (14) and (17). A drawback of this solution would be that noise would as well be amplified and this could drive the low-level system closer to instability. Furthermore, a real zero tracking error would still not be achieved. Instead, in order to improve the convergence, the estimated wrench $\hat{\mathbf{w}}_{R}$ are fed back to the low-level controller as an additional term in (12). Hereby, even the contact free flight tracking is improved as any steady state error is driven to zero (see Figure 5). More in detail, the final control input is given by

$$
\mathbf{u}=\mathbf{J}_{R}^{-1}(\alpha, \beta)\left(-\mathbf{g}\left(\boldsymbol{\omega}_{R}\right)+\mathbf{v}-\left[\begin{array}{c}
\hat{\mathbf{f}}_{R} \\
\hat{\boldsymbol{\tau}}_{R}^{R}
\end{array}\right]\right) .
$$

In case of perfect compensation of both the interaction forces and torques, the error dynamics is the same of (14) and 

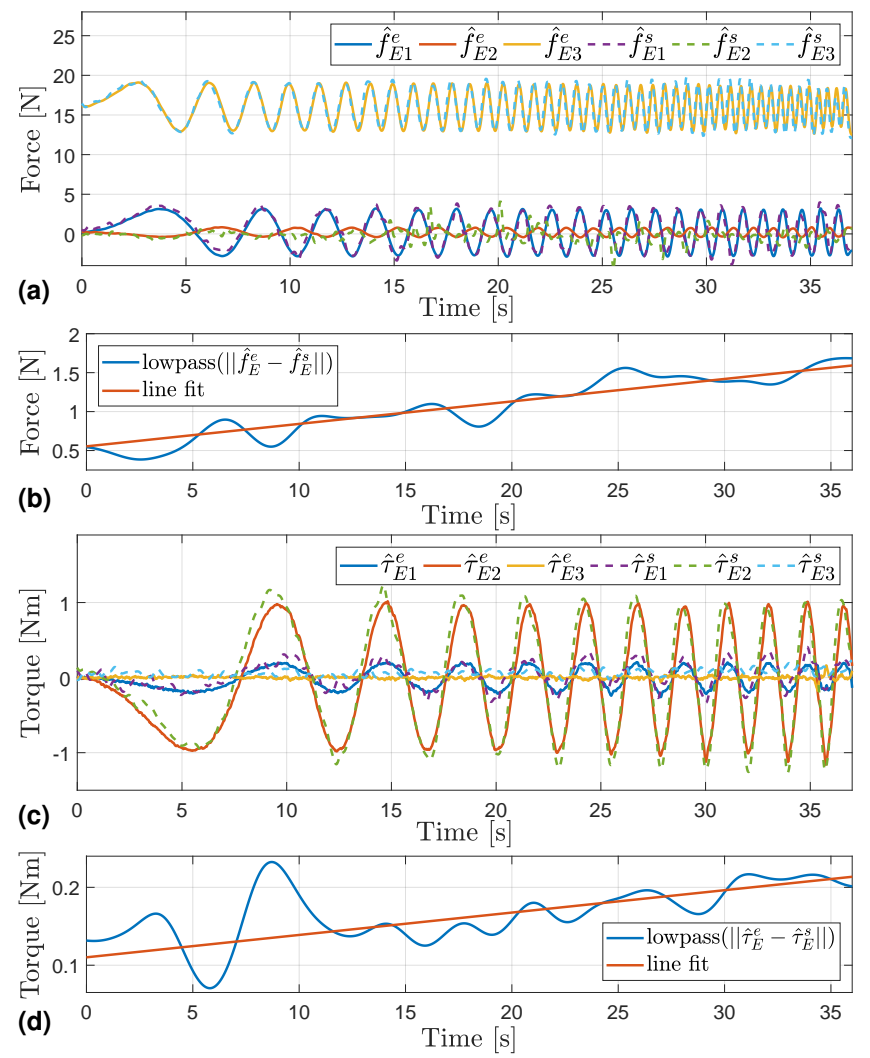

Figure 4. Estimated versus measured interaction wrench. The ground truth (dashed) is measured with an ATI45 force torque transducer. The aerial robot is rigidly connected with the sensor and simultaneously performs a translational chirp signal along the bodyframe $x$ - and $z$-axis and a rotational chirp about the $y$-axis with a peak frequency of $2.5 \mathrm{~Hz}$. Both, the estimated and the ground truth signals have been filtered with a non-causal low-pass filter with a $6 \mathrm{~Hz}$ cut-off frequency. a) Estimated (solid) against sensor-measured (dashed) forces. b) Low pass filtered norm of difference of the estimated and the measured forces (blue). The difference increases monotonically with an increasing oscillation frequency indicated by the line fit (red). c) Estimated (solid) against sensor-measured (dashed) torques. d) Low pass filtered norm of difference of the estimated and the measured torques (blue). Again the difference increases monotonically with an increasing oscillation frequency indicated by the line fit (red).

(17) and, thus, the same stability properties hold. Otherwise, under the trivial assumption that the interaction wrench is bounded, the wrench estimation error can be viewed as a bounded term as well. In (Franchi et al. 2018) the stability properties of the proposed controller, in absence of interaction, have been studied. In detail, it has been proven that, provided that the reference orientation is feasible for the TiltHex platform, the tracking errors exponentially converge to zero under mild conditions on the initial angular velocity error $\mathbf{e}_{\omega}$. Thus, by recurring to the theory of stability of perturbed systems (Khalil 2002) in the presence of nonvanishing perturbations, it is possible to state that, in the presence of bounded wrench estimation errors, the tracking error is ultimately bounded. Moreover, if the interaction wrench is constant, the wrench estimation error is convergent to zero, and, thus, after it vanishes also the tracking error will converge to zero as well.

\section{Admittance Filter}

In order to achieve bounded forces exchanged with the environment, a compliant behavior could be enforced between the position and orientation of the end-effector and the interaction generalized forces.

Assigned a planned desired trajectory of the end-effector in terms of position $\mathbf{p}_{E, d}$, orientation $\mathbf{R}_{E, d}$, velocities $\boldsymbol{v}_{E, d}=$ $\left[\dot{\mathbf{p}}_{E, d}^{\mathrm{T}} \mathbf{R}_{E, d} \boldsymbol{\omega}_{E, d}^{\mathrm{T}}\right]^{\mathrm{T}}$, and accelerations $\dot{\boldsymbol{v}}_{E, d}$, the corresponding set of reference motion variables to be fed to the motion controller, $\left(\mathbf{p}_{E, r}, \mathbf{R}_{E, r}, \boldsymbol{v}_{E, r}, \dot{\boldsymbol{v}}_{E, r}\right)$, can be generated via an admittance filter, characterized by the following dynamics

$$
\mathbf{M}_{E} \Delta \dot{\boldsymbol{v}}_{E}+\mathbf{D}_{E} \Delta \boldsymbol{v}_{E}+\mathbf{K}_{E} \mathbf{e}_{E}=\hat{\mathbf{w}}_{E}
$$

where $\Delta \boldsymbol{v}_{E}=\boldsymbol{v}_{E, d}-\boldsymbol{v}_{E, r}$ is the velocity error, while $\mathbf{e}_{E}$ is the pose error given by

$$
\mathbf{e}_{E}=\left[\begin{array}{c}
\mathbf{p}_{E, d}-\mathbf{p}_{E, r} \\
\frac{1}{2}\left(\mathbf{R}_{E, d} \mathbf{R}_{E, r}^{\mathrm{T}}-\mathbf{R}_{E, r} \mathbf{R}_{E, d}^{\mathrm{T}}\right)^{\vee}
\end{array}\right] .
$$

The (27) represents the dynamics of a 6-DoF mechanical impedance (Siciliano et al. (2009)) of inertia $\mathbf{M}_{E}$, damping $\mathbf{D}_{E}$ and stiffness $\mathbf{K}_{E}$ : those matrices are all positive-definite and suitably chosen in a way to impose an over-damped behavior to the system. Moreover, in order to guarantee the stability of the overall system, the gain matrices must ensure that the motion controller (inner loop) is characterized by a faster dynamics with respect to the admittance filter.

Once the reference trajectory of the end-effector has been computed it should be expressed in terms of CoM reference trajectory in order to be tracked by the inner loop pose controller. The reference position and orientation of the robot are then computed (see Figure 2) as

$$
\left\{\begin{array}{l}
\mathbf{p}_{R, r}=\mathbf{p}_{E, r}-\mathbf{R}_{R, r} \mathbf{p}_{E}^{R} \\
\mathbf{R}_{R, r}=\mathbf{R}_{E, r} \mathbf{R}_{R}^{E}
\end{array}\right.
$$

while the CoM reference velocities and accelerations are obtained by taking the time derivatives of (29). In detail, the reference velocities are given by

$$
\left\{\begin{array}{l}
\dot{\mathbf{p}}_{R, r}=\dot{\mathbf{p}}_{E, r}-\mathbf{R}_{R}\left[\boldsymbol{\omega}_{R, r}\right]_{\times} \mathbf{p}_{E}^{R}, \\
\boldsymbol{\omega}_{R, r}=\boldsymbol{\omega}_{E, r},
\end{array}\right.
$$

while the reference accelerations are

$$
\left\{\begin{array}{l}
\ddot{\mathbf{p}}_{R, r}=\ddot{\mathbf{p}}_{E, r}-\mathbf{R}_{R}\left[\dot{\boldsymbol{\omega}}_{R, r}\right]_{\times} \mathbf{p}_{E}^{R}-\mathbf{R}_{R}\left[\boldsymbol{\omega}_{R, r}\right]_{\times}^{2} \mathbf{p}_{E}^{R}, \\
\dot{\boldsymbol{\omega}}_{R, r}=\dot{\boldsymbol{\omega}}_{E, r}
\end{array}\right.
$$

Remark The admittance approach has been preferred to an impedance one since it allows to better counteract the model uncertainties and to separate the impedance control action from the motion control action, which can be made purposefully stiff so has to enhance disturbance rejection and ensuring good tracking performance in free space (Villani and De Schutter 2008). The motion controller described above is characterized by a bandwidth wide enough to guarantee the stability of the inner/outer loop and at same time guarantee high performance in the free space motion. Moreover, even if the environment is rigid, the flying platform and the considered tool ensure a certain level of passive compliance which confers robustness to the scheme. 


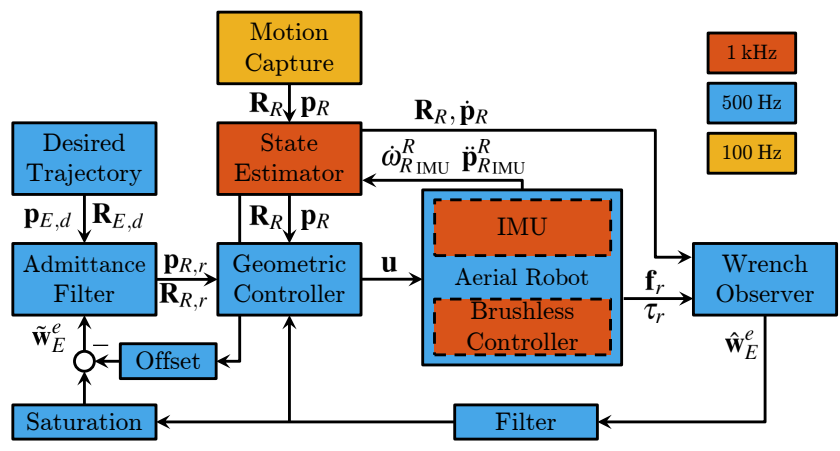

Figure 5. Signal block diagram of the control framework. The runtime frequency is highlighted. For clarity higher derivatives of the signals have been omitted.

\section{Practical Implementation}

To further improve the control scheme several adjustments have been made to enable a better performance of the system in flight and during contact.

- Modeling errors (or, more precisely, errors in the manufacturing of the aerial robot with respect to the desired model) do cause a constant error in $\hat{\mathbf{w}}_{R}$. To eliminate this error, the steady state error has been estimated during a contact-free hovering flight. Such offset has been then taken into account both in the wrench observer and in the controller.

- To suppress nonexistent small force and torque estimations due to sensor noise, we implemented a dead zone on the admittance filter input. Any norm value of force component below $0.2 \mathrm{~N}$ and any norm value torque component below $0.2 \mathrm{Nm}$ will be neglected. To achieve a continuous wrench signal the same thresholds are subtracted from higher estimations. This implies that the admittance filter receives as input a wrench slightly lower than the real interaction wrench.

- The estimated contact force $\hat{\mathbf{w}}_{E}$ is filtered with a digital lowpass-filter before it is further used.

\section{Experimental Setup}

\section{Hardware}

The Tilt-Hex robot is a LAAS-CNRS in house developed fully actuated aerial robot. All used structural components are either off-the-shelf available or 3D printable by a standard fused deposition modeling printer. The diameter of the aerial robot from rotor hub to rotor hub spans $0.8 \mathrm{~m}$. The total mass, including a $2.2 \mathrm{Ah}$ LiPo-battery and the rigid end-effector accumulates to $1.8 \mathrm{~kg}$. The tool-tip of the endeffector is placed off-centered from the robot's center of mass by $\mathbf{p}_{E}^{R}=\left[\begin{array}{llll}0.12 & 0 & 0.4\end{array}\right]^{\mathrm{T}} \mathrm{m}$. The inertia tensor is estimated by a high detail CAD model and the principal components are identified as $\mathbf{J}=\operatorname{diag}(11.5,11.4,19.4) 10^{-6} \mathrm{~kg} \mathrm{~m}^{2}$. To achieve the full actuation the propellers are tilted about two axes $\alpha=30^{\circ}$ and $\beta=10^{\circ}$. As indicated by the \pm every second motor-propeller combination is tilted about a negative $\alpha$-angle. The particular choice of $\alpha$ and $\beta$ is a compromise between maximum lateral forces and a minimization wasted internal forces (Rajappa et al. (2015)). The speed of each propeller is accurately regulated and measured by using the algorithm and hardware presented in Franchi and Mallet (2017).

To retrieve the Tilt-Hex's pose estimation and its derivatives the aerial robot contains a standard IMU with accelerometers and gyroscopes, providing the sensor information at $500 \mathrm{~Hz}$. The on-board micro-controller is only used for the data transmission of the IMU readings and the motor commands. Furthermore, optical markers are attached on the Tilt-Hex utilized by an external motion capture system (Optitrack MoCap) providing position and orientation information at $100 \mathrm{~Hz}$ (notice that a PnP algorithm and an onboard camera could straightforwardly replace the MoCap in this case). The on-board and external sensor information are fused by an UKF state estimator, providing full state estimation at $500 \mathrm{~Hz}$.

To validate the contact wrench estimator a $3 \mathrm{D}$ force torque sensor (ATI Mini45) has been used off-line to validate the estimation results. By using the sensor, we have been enforced by our decision to use an estimator instead of a sensor on board as the sensor plus the electronics reduces the payload and suffers strongly from temperature drift in even short time horizons.

\section{Software}

The full control framework described above has been implemented in a Matlab Simulink environment to boost a fast development. The controller (see Figure 5) runs at $500 \mathrm{~Hz}$ in soft real-time on a standard desktop machine. For safety the aerial robot is currently connected via a serial connection (RS232) with the desktop machine, which transmits the desired propeller spinning velocities to the TiltHex and the actual propeller spinning velocity, IMU data, all at $500 \mathrm{~Hz}$, and further status updates, e.g., safety checks, battery status with a lower frequency to the base PC. In the future we plan in a first step to replace the serial cable with a powerful wireless serial connection, while on the long run the control shall be exported to $\mathrm{C}$ and run on the aerial robot itself. The MoCap measurements $(100 \mathrm{~Hz})$ are fused via a UKF state estimator with the IMU measurements $(500 \mathrm{~Hz})$ thus obtaining a full state estimate at $500 \mathrm{~Hz}$.

\section{Experimental Results}

To present the capabilities and limits of the control framework a broad spectrum of different experiments has been conducted (see Figure 6) - the interested reader is as well referred to the attached multimedia data. Firstly, we will present a bench of experiments demonstrating the physical property shaping capabilities of the outer loop admittance filter. Secondly, we show a hard contact and sliding of the off-centered tool-tip while making use of the afore presented physical property shaping to fulfill a desired task. Thirdly we present a challenging flying peg-in-hole task. Finally, sliding on a surface mounted on a force/torque sensor is proposed in such a way to show the effectiveness of the wrench estimate also in the presence of time-varying forces. Where needed, we will indicate the components of a generic vector $[\bullet] \in \mathbb{R}^{3}$ by the letters $x, y$ and $z$, i.e., $[\bullet]=\left[[\cdot]_{x}[\cdot]_{y}[\cdot]_{z}\right]^{T}$. 


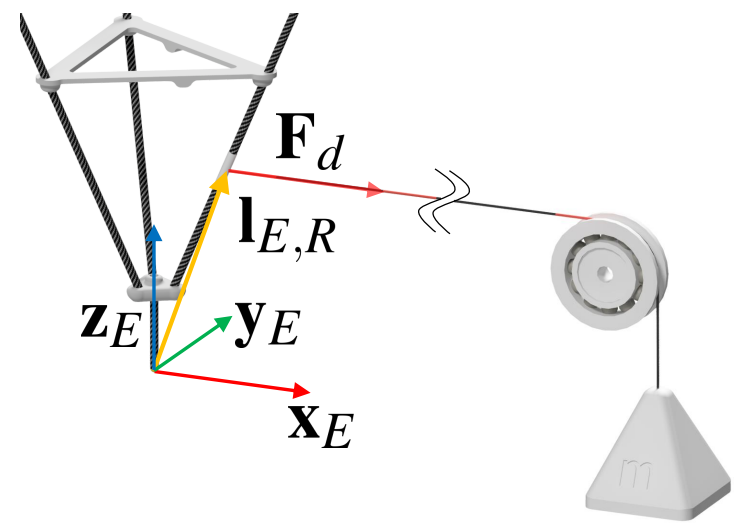

a)

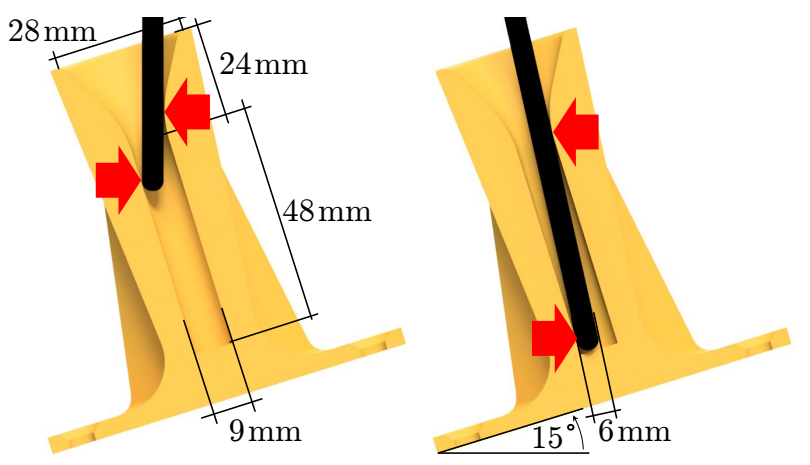

c)

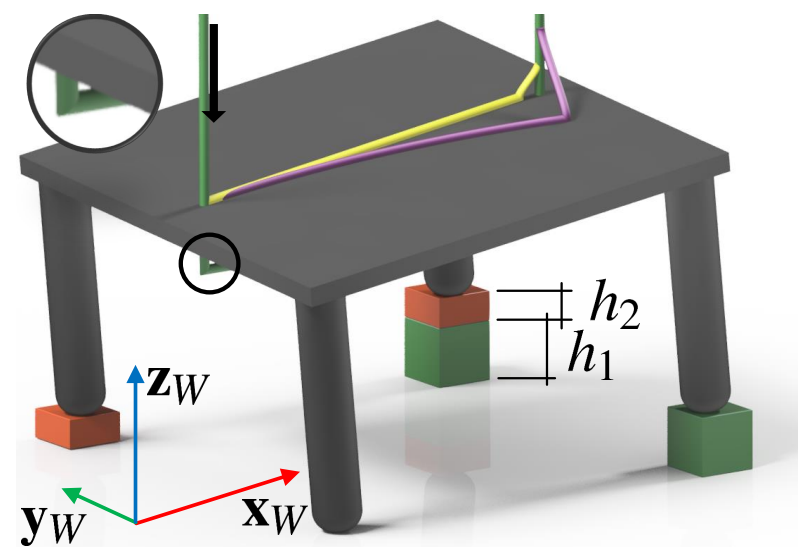

b)

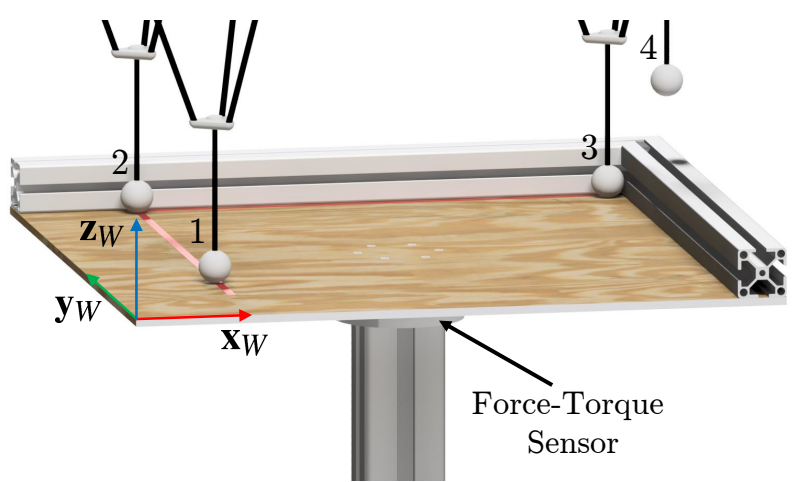

d)

Figure 6. Setups of the four conducted experiments: a) Left side shows the end-effector of the aerial robot. A mass pulls on a rope fixated on the end-effector, resulting in a force $\mathbf{F}_{d}$ in the direction of $\mathbf{x}_{W}=\mathbf{x}_{E}$. The force is applied offsetted by $\mathbf{l}_{E, R}$ with respect to the tool-tip. b) Sliding with constant force application on a rigid, tilted surface. The black arrow indicates the direction of the trajectory. The green line represents the desired trajectory $\mathbf{p}_{E, d}$, which pierces the tilted surface (magnification shows the reference trajectory below the table). The yellow and violet lines represent the two reference trajectories, due to the contact forces, generated by two different values of the admittance parameter $\mathbf{K}_{E}$. c) Peg in hole task with the tool-tip into a funnel. Left side illustrates the moment of contact, the right side illustrates the adapted and fully inserted tool-tip. The funnel is tilted by $15^{\circ}$ but the desired trajectory orientation $\mathbf{R}_{E, d}$ assumes a vertical funnel. Due to the contact forces (indicated by the red arrows) the admittance filter reconfigures the reference orientation $\mathbf{R}_{E, r}$ and allows for the pegging. d) Sliding with multiple contacts: The end-effector slides along the surface from the initial contact point (numbered with 1) to the final point (indicated with 3 ) along the red line. The numbers correspond to the numbers in Figure 13 and indicate where the phases change. 1 indicates a single contact, 2 indicates two contacts, 3 indicates three contacts, with no lateral motion possible and 4 indicates free-flight.

\begin{tabular}{|c|c|c|c|c|}
\hline & & Sliding 1 & Sliding 2 & Peg-In-Hole \\
\hline \multirow{6}{*}{$\begin{array}{c}\text { Admit- } \\
\text { tance } \\
\text { Filter }\end{array}$} & $\mathbf{M}_{E_{P}}$ & 1.5 & 1.5 & 1.5 \\
\hline & $\mathbf{D}_{E_{P}}$ & {$\left[\begin{array}{llll}10 & 3.5 & 10\end{array}\right]$} & {$\left[\begin{array}{lll}10 & 10 & 10\end{array}\right]$} & 10 \\
\hline & $\mathbf{K}_{E_{P}}$ & {$\left[\begin{array}{llll}10 & 2.5 & 6\end{array}\right]$} & {$\left[\begin{array}{lll}10 & 10 & 6\end{array}\right]$} & 6 \\
\hline & $\mathbf{M}_{E_{R}}$ & {$\left[\begin{array}{lll}1 & 1 & 2\end{array}\right] \cdot 10^{-2}$} & {$\left[\begin{array}{lll}1 & 1 & 2\end{array}\right] \cdot 10^{-2}$} & {$\left[\begin{array}{lll}1 & 1 & 2\end{array}\right] \cdot 10^{-2}$} \\
\hline & $\mathbf{D}_{E_{R}}$ & 4 & 4 & 4 \\
\hline & $\mathbf{K}_{E_{R}}$ & {$\left[\begin{array}{lll}10 & 10 & 5\end{array}\right]$} & {$\left[\begin{array}{lll}10 & 10 & 5\end{array}\right]$} & {$\left[\begin{array}{llll}8 & 5 & 35\end{array}\right]$} \\
\hline
\end{tabular}

Table 2. Parameters of the admittance filter in (27) in the two surface sliding tasks and in the peg-in-hole task. A single value represents the multiplier of a $3 \times 3$ identity matrix. The changing parameters in the two sliding experiments are highlighted in bold. A vector represents the entries of a $3 \times 3$ diagonal matrix.

\section{Physical properties shaping}

We will now experimentally test and demonstrate the physical property shaping capabilities of the outer loop admittance filter with respect to the end-effector tool-tip. By exerting a step-like force profile on the aerial robot we will show that we can achieve a large variety of desired massspring-damper behaviors.

During all these experiments the desired end-effector position, $\mathbf{p}_{E, d}=\left[\begin{array}{lll}0 & 0 & 1\end{array}\right]^{T} \mathrm{~m}$, and orientation, $\mathbf{R}_{E, d}=\mathbf{I}$, in (27) are kept constant over time. During steady state hovering of the aerial robot a step disturbance force is applied. The disturbance force is unaligned with the tool-tip by the vector $\mathbf{l}_{E, R}$ (see Figure 6a), resulting not only in a force but as well a torque with respect to the tool-tip. The disturbance step is realized by a released mass $(m=0.14 \mathrm{~kg})$ pulling on a taut cable along $\mathbf{x}_{E}$, fixated on the end-effector of the aerial robot. Resulting in a pulling disturbance force of about $\mathbf{F}_{d}=\left[\begin{array}{lll}1.4 & 0 & 0\end{array}\right]^{T} \mathrm{~N}$. Once the aerial robot reaches a new steady state hovering the force is removed by lifting the weight and keeping the cable slack. To demonstrate the full capabilities of the admittance filter, the three parameters $\mathbf{M}_{E}, \mathbf{D}_{E}$ and $\mathbf{K}_{E}$ in (27) have been individually modified. As reference values we chose $\mathbf{M}_{E}=1.5 \mathbf{I}_{3} \mathrm{~kg}, \mathbf{D}_{E}=10 \mathbf{I}_{3} \mathrm{~kg} / \mathrm{s}$ and $\mathbf{K}_{E}=$ 
$6 \mathbf{I}_{3} \mathrm{~kg} / \mathrm{s}^{2}$. In three experimental batches we increased and decreased each of the three parameters while keeping the other two unchanged.

Firstly we changed the spring constant to $\mathbf{K}_{E}=4 \mathbf{I}_{3} \mathrm{~kg} / \mathrm{s}^{2}$ and $\mathbf{K}_{E}=8 \mathbf{I}_{3} \mathrm{~kg} / \mathrm{s}^{2}$, see Figure $7 \mathrm{a}$. To allow an evaluation of the results we simulated additionally an ideal massspring-damper system (msd-system) under the same force disturbance and compared the position output $p_{E, i_{x}}$ of the ideal msd-system with the reference position output $\mathbf{p}_{E, r}$ in (28) of a real experiment. The results show firstly that an ideal msd-system is emulated very well by our admittance scheme as the ideal trajectory is tracked precisley. The maximum position difference between the reference position and the msd-system $\left(p_{E, i_{x}}-p_{E, r_{x}}\right)$ is below $0.02 \mathrm{~m}$ for $\mathbf{K}_{E}=$ $6 \mathbf{I}_{3} \mathrm{~kg} / \mathrm{s}^{2}$. Secondly, as desired, the modification of the spring constant results in three different behaviors and different final steady state positions $(0.14 \mathrm{~m} 0.19 \mathrm{~m} 0.29 \mathrm{~m})$. These results match very well the expected steady state positions $(0.15 \mathrm{~m} 0.20 \mathrm{~m} 0.30 \mathrm{~m})$ considering the utilized dead-zone (see Sec. Practical Implementation). Every single trial is tracked well. Thirdly, it is worth to note the differences in charging and discharging of the spring. The reproduction of an ideal msd-system is much better mirrored during discharging - as well the ideal trajectory is better tracked. We assume this is due to small but immanent friction and stick-slip effects that are in-existent during discharging. To give more insides of the msd-system we plotted the first component of the low level position error $\mathbf{e}_{p}=\left[e_{p_{x}} e_{p_{y}} e_{p_{z}}\right]^{T}$ (see (14)) in Figure 7-d for the three different $\mathbf{K}_{E}$. The largest position error occurs shortly $(<1 \mathrm{~s})$ after the force application. This is explained by the nature of the wrench observer, which suppresses high frequency signals. Figure 7e presents the velocity profile along the first component of the velocity vector $\dot{\mathbf{p}}=\left[\begin{array}{lll}\dot{p}_{x} & \dot{p}_{y} & \dot{p}_{z}\end{array}\right]^{T}$ which coincides with the direction of the applied force. It is nicely visible that changing $\mathbf{K}_{E}$ has only minimal effect on the velocity. Last Figure $7 \mathrm{f}$ show the first component of the wrench estimator acting on the tool-tip as in (25). It is clear that the estimated forces overlap very well in all cases, which is a further demonstration of the good performance of the estimator.

In the second experimental batch the damping constant was changed to $\mathbf{D}_{E}=5 \mathbf{I}_{3} \mathrm{~kg} / \mathrm{s}$ and $\mathbf{D}_{E}=20 \mathbf{I}_{3} \mathrm{~kg} / \mathrm{s}$ while $\mathbf{K}_{E}$ was set back to its initial value. In contrast to the first experimental batch the steady state value is now identical in all three cases since it depends only on $\mathbf{K}_{E}$. To get a better insight of the effect of a changed damping parameter we now compared the ideal msd-system velocity $\dot{\mathbf{p}}_{E, i}$ with the real reference velocity output $\dot{\mathbf{p}}_{E, r}$ in Figure $7 \mathrm{~b}$. The actual velocity tracks the reference velocity of the msdmodel although a time delay is recognizable. The delay is an expected result of the wrench estimator. Again the charging process differs from the discharging process, which appears to be much smoother.

Finally, in the last experimental batch we compared the influence of a changed mass property $\mathbf{M}_{E}$ comparing the nominal mass $\mathbf{M}_{E}=1.5 \mathrm{~kg}$ with a reduced mass of $\mathbf{M}_{E}=0.75 \mathrm{~kg}$ and an increased mass of $\mathbf{M}_{E}=3 \mathrm{~kg}$. The results are not as obvious as in the previous experiments (see Figure 7c). The ideal trajectory in this case has an instantaneous acceleration response to the applied force. Comparing the results in Figure $7 \mathrm{c}$ it turns out that the actual acceleration response is slower and lower than expected from the ideal msd-system. This behavior is explained by the first order low-pass dynamics of the wrench observer. Nevertheless the highest simulated mass $\mathbf{M}_{E}$ results, as expected, in the smallest acceleration and vice versa.

\section{Sliding on Surface}

In this experiment, we conducted a hard contact between the tool-tip of the rigid end-effector and a tilted wooden surface (see Figure 6b). The surface is tilted about $\mathbf{x}_{W}$ by $10^{\circ}$ and about $\mathbf{y}_{W}$ by $-10^{\circ}$. For a better understanding the tilting of the surface is visualized by orange and green colored bricks in Figure 6b. The preplanned translational trajectory consists out of five phases. First the desired trajectory approaches without contact from an initial position to a position $0.14 \mathrm{~m}$ above the surface (approaching phase). Then it descends from a height of $0.6 \mathrm{~m}$ to $0.35 \mathrm{~m}$ while piercing through the surface (initial contact point with the surface at $0.46 \mathrm{~m}$ ) with $0.05 \mathrm{~m} / \mathrm{s}$ (contacting phase). The desired trajectory then translates along the $\mathbf{x}_{W}$-axis for $0.4 \mathrm{~m}$ with a peak velocity of $0.12 \mathrm{~m} / \mathrm{s}$ while the other two axes remain constant (sliding phase). After the lateral motion stops the reference trajectory lifts off to its initial height, resulting in a release of the contact (release phase). Finally the desired trajectory achieves a stopping position without any further physical contact (departing phase). To reduce stick slip effects between the tool-tip and the surface during the sliding-phase the desired orientation of the tool-tip is tilted forward by $\left(\theta_{R, d}=7.5^{\circ}\right)$. To demonstrate the influence of the spring gain $\mathbf{K}_{E}=\operatorname{diag}\left[10 K_{E_{y}} 5\right]$ and the usefulness of its tuning with respect to the task at hand, the experiment has been conducted twice - first with $K_{E_{y}}=2.5 \mathrm{~kg} / \mathrm{s}^{2}$ and then with $K_{E_{y}}=10 \mathrm{~kg} / \mathrm{s}^{2}$. An illustrative snapshot series of the experiment is depicted in Figure 9.

The results of the first experiment $\left(K_{E_{y}}=2.5 \mathrm{~kg} / \mathrm{s}^{2}\right)$ are visualized in Figure 8. To simplify the understanding of the plots, the contacting phase and sliding phase have been highlighted with a green background, bordered by dashed black lines in all plots. The gray dashed line indicates the moment when the sliding phase starts. Let us first discuss the position results. Plot $8 \mathrm{a}$ presents the three components of the robot's desired position $\mathbf{p}_{R, d}$ (dashed lines) versus the output of the admittance filter, namely the three components of the reference position $\mathbf{p}_{R, r}$ (doted dashed lines) and the components of actual robot position $\mathbf{p}_{R}$ (solid lines). During the approaching phase the single components of the desired, reference and actual trajectory overlap perfectly. Starting from the contacting phase the z-component of the reference trajectory (doted dashed blue line) starts to diverge from the desired trajectory (red dashed line). The desired trajectory pierces the surface, while the reference position remains on the surface of the contact due to the sensed force (see plot $8 \mathrm{c}$ ). Plot $8 \mathrm{~b}$ illustrates the aerial robot's position error $\left(\mathbf{e}_{p}=\mathbf{p}_{R, r}-\mathbf{p}_{R}\right)$. The norm of the position error $\left|\mathbf{e}_{p}\right|$ remains marginal, below $2 \mathrm{~cm}$, during all phases of the experiment. Thus the tool-tip is perfectly tracking the reference trajectory. Plot $8 \mathrm{c}$ presents a low-pass filtered output of the first three components of the wrench observer as in (25), namely the estimated forces $\hat{f}_{E_{1}}, \hat{f}_{E_{2}}$ and $\hat{f}_{E_{3}}$. The moment of contact establishment is easily recognizable with a peak force in $\hat{f}_{E_{3}}$ 


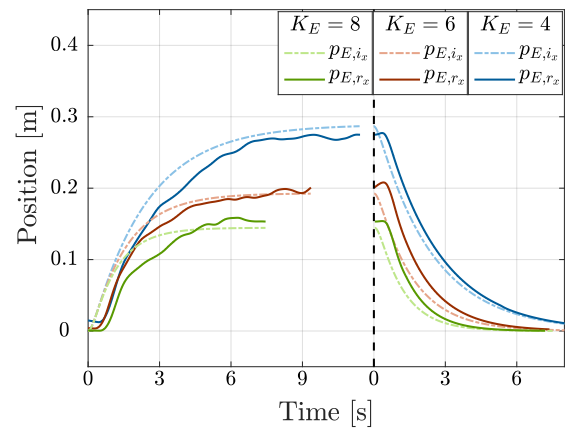

a)

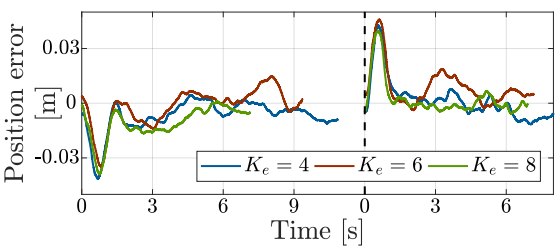

d)

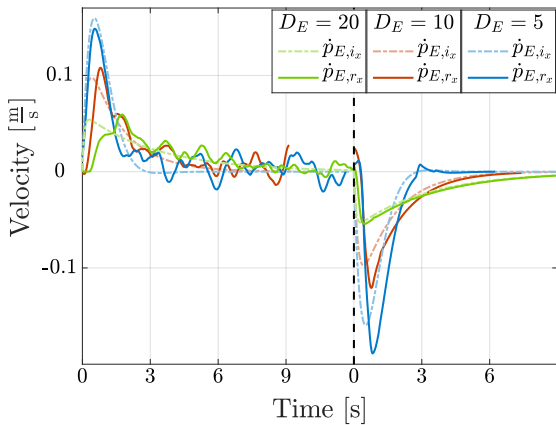

b)

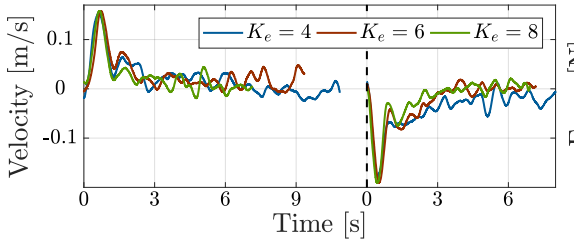

e)

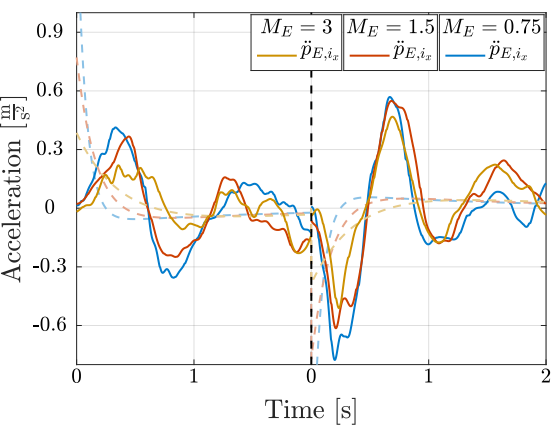

c)

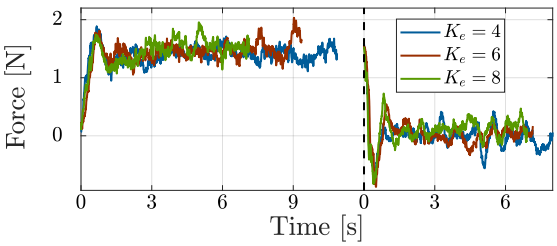

f)

Figure 7. Physical property shaping: During this experiment the aerial robot is charged with a constant force disturbance, at the dashed line the force is removed. For more clarity the time is restarted in the moment of force removal in all plots. The force is applied along $\mathbf{x}_{E}$ therefore only the first component of the position or velocity vector is presented in all plots (solid lines). The doted dashed dotted lines shows an ideal mass spring damper system. a) The spring constant $\mathbf{K}_{E}$ in (27) is varied, resulting in different steady state positions. b) The damping constant $\mathbf{D}_{E}$ is varied. The steady state position is constant in all three cases but velocity differs significantly. c) The mass constant $\mathbf{M}_{E}$ is varied, resulting in different rising times. For better visualization the x-axis scaling is different than before.

Last three plots: Further data of experiment Sec. Physical properties shaping. d) Low level position error along $p_{x}$ as $\mathbf{e}_{p}$ in (14).

e) Velocity component along $\dot{p}_{x}$. f) Force estimate $\hat{f}_{E_{1}}^{e}$ identical with the first component of $\hat{\mathbf{w}}_{E}$ in (25).

(yellow line). Starting from the sliding phase (vertical dashed gray line), smaller forces $\hat{f}_{E_{1}}$ (blue line) and $\hat{f}_{E_{2}}$ (red line) are estimated as well. $\hat{f}_{E_{1}}$ is the force opposing the sliding direction resulting from the increased slope and friction effects between the tool-tip and the surface. The force $\hat{f}_{E_{2}}$ is a result of the tilted surface and the contact force $\hat{f}_{E_{3}}$. Without any position controller the tool-tip would slide down the tilted surface. The fourth plot (8d) presents the difference between the components of the preplanned desired position trajectory and the admittance filter output $\left(\mathbf{p}_{R, d}-\mathbf{p}_{R, r}\right)$. It shows the deflection of the reference trajectory due to the sensed forces $\hat{f}_{E}$. Plots $8 \mathrm{e}$ to $8 \mathrm{~h}$ present the orientation results of the same experiment. During the approaching phase the aerial robot's desired pitch orientation changes from $\theta=0^{\circ}$ to $\theta=7.5^{\circ}$ and remains constant hereafter. The pitching angle is chosen to achieve a better sliding. During the contact free phase the reference orientation follows the desired orientation perfectly as only negligible torques are sensed (see last plot $8 \mathrm{~h}$ ). As well the tracking error between the reference orientation and the actual orientation (see $\mathbf{e}_{R}$ in plot 8f) remains very small. During the sliding phase a non-negligible torque $\hat{\tau}_{E_{2}}$ is estimated, resulting in an additional pitching of more than $2.5^{\circ}$ and an total pitch angle $\theta_{R}$ of $10^{\circ}$ of the aerial robot. Furthermore the tilting of the surface about $\mathbf{x}_{W}$ causes an additional small adaptation of the reference roll trajectory (see plot $8 \mathrm{~g}$ ).

Now we want to make use of the property shaping capabilities of the admittance filter. In a fictitious task it is desirable that the aerial robot is compliant along the $\mathrm{z}$ axis but stiff along $\mathrm{x}$ and $\mathrm{y}$ and should therefore not glide down the slope of the tilted surface towards $-\mathbf{x}_{y}$. In the first experiment, the tool-tip diverged from $p_{R, d_{y}}$ by $\approx 0.1 \mathrm{~m}$. To achieve a more desirable behavior we increase the gain $K_{E_{y}}$ in (27) from 2.5 to 10 . The result and the original values are plotted in Figure 10. It becomes directly clear, that the aerial robot follows a very similar trajectory as before in $\mathrm{x}$ and $\mathrm{z}$ direction. In $\mathrm{y}$ direction the aerial robot slid down the slope before. In the second experiment the tool-tip is much stiffer in y direction and does almost not diverge. The difference between the desired and the reference trajectory $\left(p_{R, d_{y}}-p_{R, r_{y}}\right)$ is almost zero at all times. Now, the tool-tip of the end-effector follows a trajectory close to the yellow example trajectory in Figure $6 b$, while before the trajectory was similar to the purple trajectory in the same figure.

\section{Peg-In-Hole task}

The experimental setup of the peg-in-hole task employs a $15^{\circ}$ tilted standing funnel. The funnel has a maximum diameter of $28 \mathrm{~mm}$ at the beginning, narrowing down into a $48 \mathrm{~mm}$-deep $9 \mathrm{~mm}$-wide tube (see Figure $6 \mathrm{c}$ ). The diameter of the end-effector is $6 \mathrm{~mm}$ - allowing a lateral play of only $\pm 1.5 \mathrm{~mm}$ once the end-effector is inserted. The preplanned trajectory expects an upright funnel $\left(\mathbf{R}_{E, d}=\mathbf{I}\right)$ and is not aware of the $15^{\circ}$ tilting. The goal of this experiment is to place the end-effector in the funnel, hold it inside and remove it safely. Hereby our expectations are twofold: firstly, we want to demonstrate the precise pose control of the endeffector, able to place the tool-tip exactly above the funnel, secondly but more interesting, we want to underline the importance of task robustness and the ability of the system to adapt to such large orientation errors thanks to the aerial 

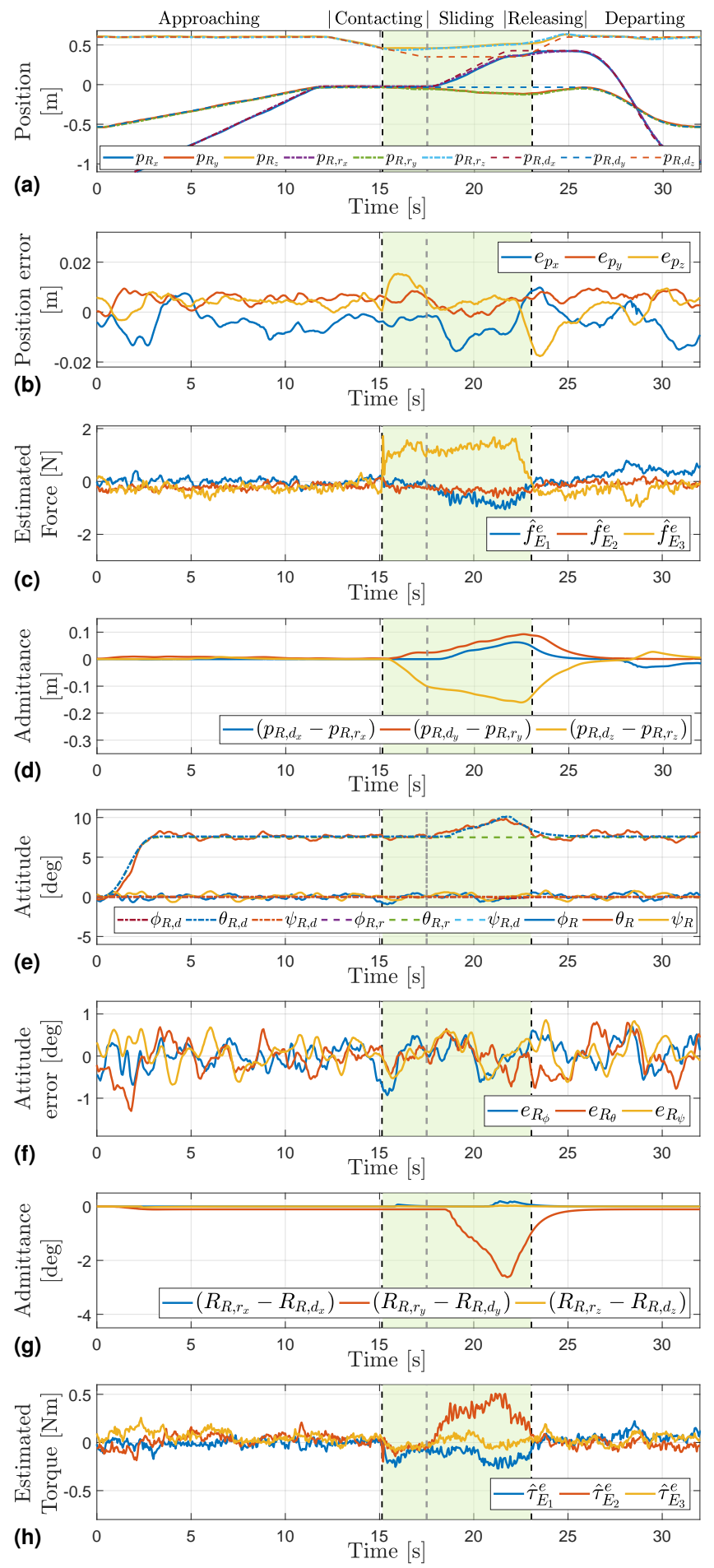

Figure 8. Sliding with the tool-tip on a tilted surface (see Figure $6 \mathrm{~b}$ ). The contact phase is highlighted in green, while the actual sliding starts at the gray dashed line: a) Desired, reference and actual position of the tool-tip. b) Actual position error between tool-tip and reference trajectory as $\mathbf{e}_{p}$ in (14).

c) Estimated tool-tip contact forces - low pass filtered (25).

d) Difference between desired trajectory $\mathbf{p}_{R, d}$ and the admittance filter output $\mathbf{p}_{R, r}$ due to the contact force. e) Desired, reference and actual tool-tip orientation. f) Actual orientation error between tool-tip and reference trajectory as in (15).

g) Difference between desired trajectory $\mathbf{R}_{R, d}$ and the admittance filter output $\mathbf{R}_{R, r}$ for convenience expressed in Euler angles. h) Estimated tool-tip contact torques - low pass filtered output of (25).
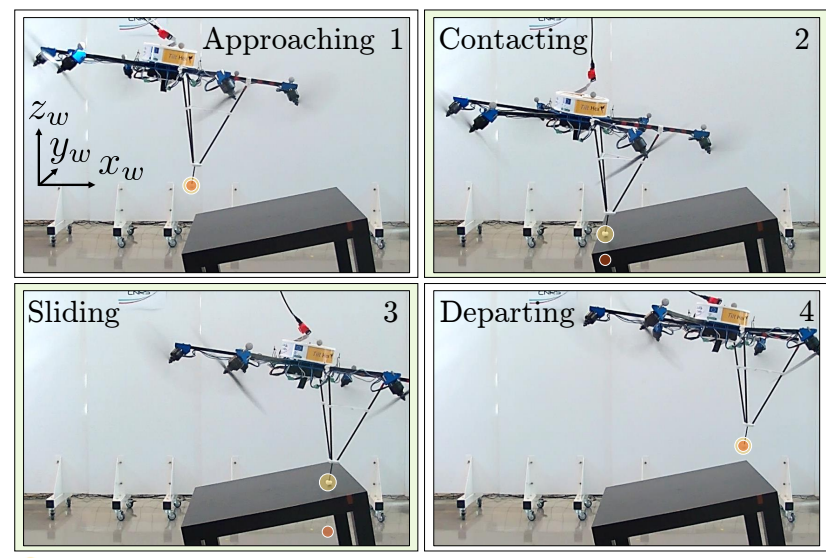

Actual end-effector position

Desired end-effector position

Figure 9. Snapshots of the Sliding task, the desired end-effector position (brown circle) and the actual position (yellow circle) are highlighted. The background colors match the colors of the contact phases in Figure 8. 1) Approaching the surface. 2.) Establishing contact with surface. The desired and actual end-effector position separate, resulting in the contact force 3.) Sliding along the tilted surface with an increasing distance between desired and actual end-effector position. 4.) Departing the surface. Desired and actual position unite again.

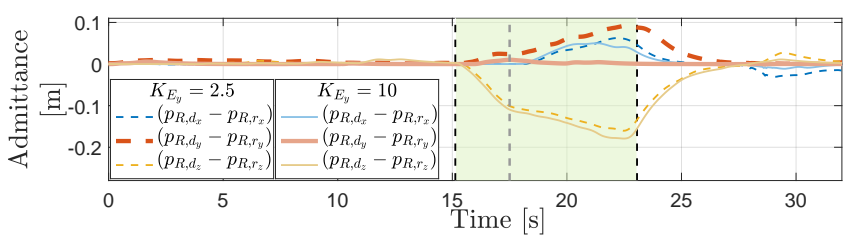

Figure 10. Comparison of the sliding experiment with a low spring gain $k_{E_{y}}=2.5$ (dashed) and a high spring gain $k_{E_{y}}=10$ (solid line). Only the spring gain along $y$ has been altered while the gains along the other two axis remains the same. In the first case the aerial robot is compliant along $y_{B}$ (red dashed line) and slides down the tilted surface by almost $0.1 \mathrm{~m}$. In the second case the tool-tip precisely follows the reference trajectory (red solid line) with negligible deviation. The other two axes $\left(x_{B}\right.$ and $\left.z_{B}\right)$ behave almost identical in the two cases.

force control. By sensing the contact torque between the tooltip and the funnel, the reference orientation shall be adapted such that the aerial robot is able to place the tool-tip into the funnel.

In detail, the desired end-effector trajectory first follows a translation along $\mathbf{x}_{W}$ and stops $0.16 \mathrm{~m}$ above the funnel (see Figure 12a). Then the aerial robot decreases the altitude along $\mathbf{z}_{W}$ and shall penetrate the tube section of the funnel with the end-effector slowly $\left(\dot{p}_{R, r_{z}}=0.01 \mathrm{~m} / \mathrm{s}\right.$ ). To do so, the aerial robot needs to change its actual pitching attitude from $\theta=0^{\circ}$ to $\theta=15^{\circ}$. Finally the funnel shall be penetrated completely.

To achieve a compliant behavior with respect to the wrong orientation of the funnel the orientation gains $\left(\mathbf{M}_{E_{R}}, \mathbf{K}_{E_{R}}\right.$ in (27)) of the admittance filter have been selected to be small (see Tab. 2). If $\mathbf{M}_{E_{R}}, \mathbf{K}_{E_{R}}$ are chosen too high then the endeffector is not entering the funnel as the rigid end-effector gets stuck.

The experimental results are reported in Figure 12. The contact between the end-effector and the funnel is established between $4.5 \mathrm{~s}$ and $16 \mathrm{~s}$ (marked by vertical bars 


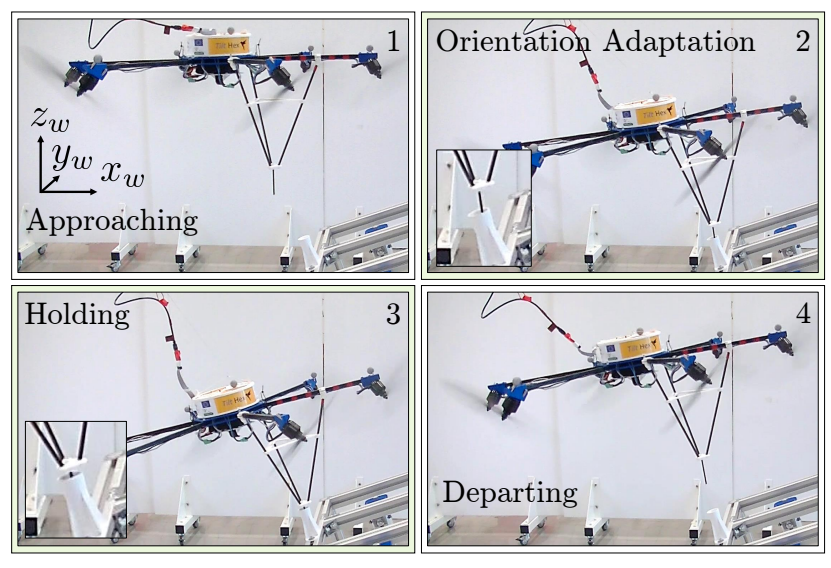

Figure 11. Snapshots of the Peg-in-Hole task, contact forces are highlighted with red arrows. The background colors match the colors of the contact phases in Figure 12. 1) Approaching the hole. 2.) Adapting attitude to peg the hole. 3.) Holding end-effector in hole. 4.) Departing and readjusting attitude to desired horizontal orientation.

in Figure 12). The contact phase has been highlighted with a green background in all plots. Starting from the moment of contact, the tool-tip is exposed to a torque, due to the two contact points (see Figure $12 \mathrm{f}$ and Figure $6 \mathrm{c}$ )). The torque causes the admittance filter to adapt the reference pitch orientation $\theta_{R, r}$ with respect to the desired angle $\theta_{R, d}$ (see Figure 12e) until the tip can be fully inserted into the funnel. Once the contact is detached the reference orientation $\theta_{R, r}$ decreases again. Furthermore we can report that the inner loop position and orientation errors remain negligible (see Figure 12b and Figure 12d).

We would like to point out that this task would be clearly unfeasible for any underactuated aerial platform because of the strong coupling between lateral motion and the vehicle's attitude.

\section{Multi contact sliding}

The last conducted experiment is inspired by common industrial tasks like inspection with contact, surface polishing or welding where, while translating, a particular force application on a surface is needed. In the experiment, the end-effector is commanded to slide along a horizontal plane and applies a dynamic force profile. At the same time, the number of translational constraints changes from one, to two, to finally three-allowing for no lateral movement. For this experiment a horizontal plate has been mounted on top of a force-torque sensor (which serves as ground truth). Additionally, two ledges aligned with $\mathbf{x}_{W}$ and $\mathbf{y}_{W}$ limit the plate (see Figure 6d and Figure 13). After establishing contact between the end-effector and the horizontal plate, the experiment consists of three phases. In the first phase, highlighted with bright green in Figures 13-1 and 14, the end-effector applies a vertical force along $\mathbf{z}_{W}$ while sliding in direction $\mathbf{y}_{W}$, until the first ledge is touched (see as well Figure 13-1). Then, the second phase starts with applying a constant force against the ledge, in direction $\mathbf{y}_{W}$ (highlighted with bright blue in Figure 14 and Figure 13-2. Next, the end-effector slides along the ledge in direction $\mathbf{x}_{W}$ and additionally a saw-tooth force profile is applied along $\mathbf{z}_{W}$,
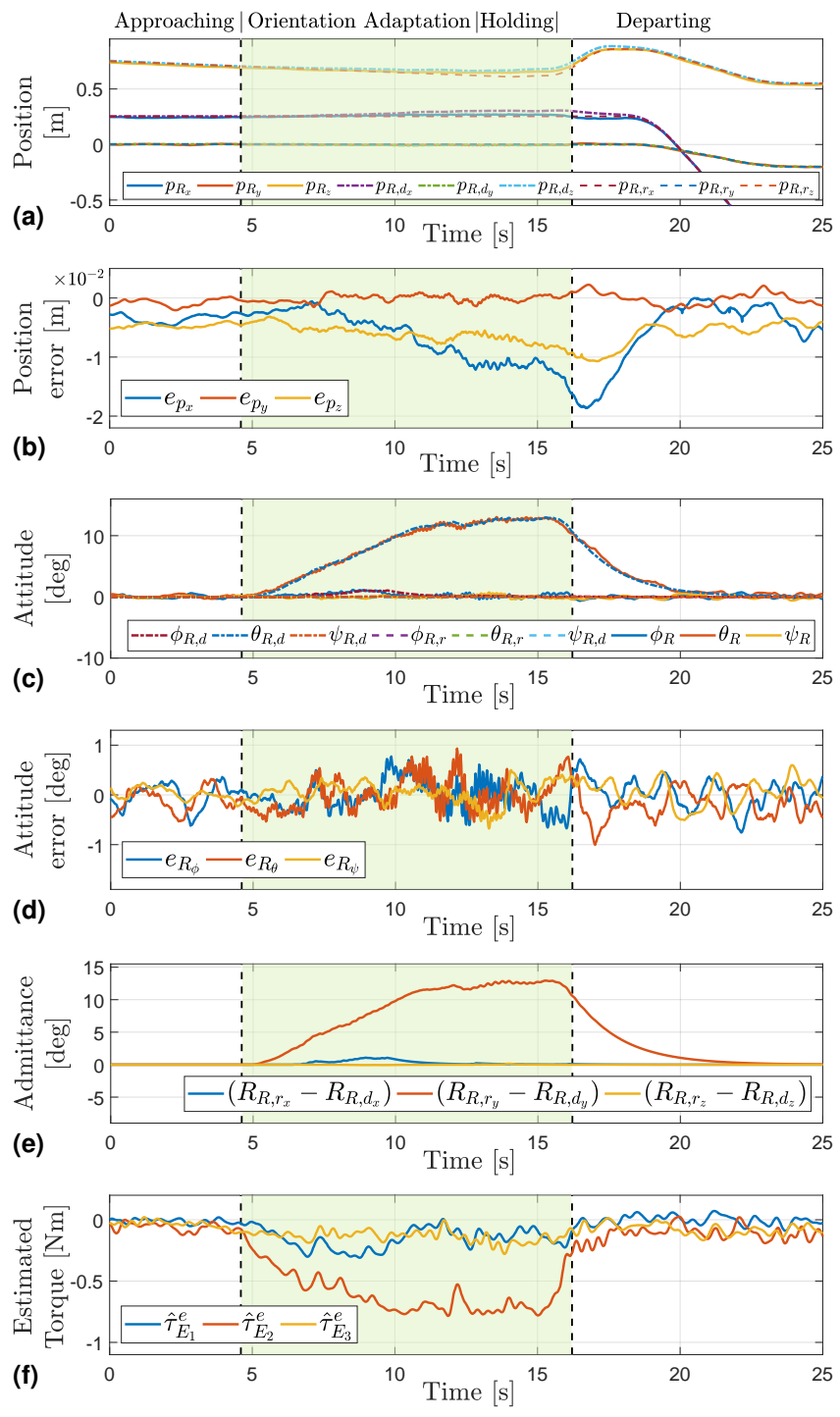

Figure 12. Flying Peg-in-Hole task with minimal lateral play. a) Reference (dashed), desired (dot-dashed) and actual position (solid). b) Position error as $\mathbf{e}_{p}$ in (14). c) Reference (dashed), desired (dot-dashed) and actual orientation (solid). d) Orientation error as defined in (15). e) Difference between desired trajectory $\mathbf{R}_{R, d}$ and the admittance filter output $\mathbf{R}_{R, r}$ for convenience expressed in Euler angles. f) Estimated tool-tip contact torques - low pass filtered output of (25).

marked with a circled 1 in Figure 14a. Once the second ledge is reached, the third phase starts (highlighted with bright red in Figure 14 and Figure 13-3. The end-effector is now in contact with three sides and cannot translate anymore. Sawtooth force profiles are now consecutively applied along $\mathbf{y}_{W}$ and $\mathbf{x}_{W}$, marked with a circled 2 and 3 in Figure 14a. Finally the hexarotor takes off and detaches the contact between the surface and the end-effector.

Figure 14 presents the experimental results. The reference $\mathbf{p}_{R, r}$, desired $\mathbf{p}_{R, d}$ and actual positions $\mathbf{p}_{R}$ are depicted in Figure 14a, showing first, an error free matching of $\mathbf{p}_{R}, \mathbf{p}_{R, r}$ and $\mathbf{p}_{R, d}$ in the absence of a contact force and second, a divergence between $\mathbf{p}_{R, d}, \mathbf{p}_{R, r}$ during contact, while the end-effector $\mathbf{p}_{R}$ still perfectly tracks $\mathbf{p}_{R, r}$. The admittance filter effect, i.e., the difference between $\mathbf{p}_{R, d}$ and $\mathbf{p}_{R, r}$ is presented in Figure 14b. It is nice to see how the three contact phases (single, double and triple) match well with 


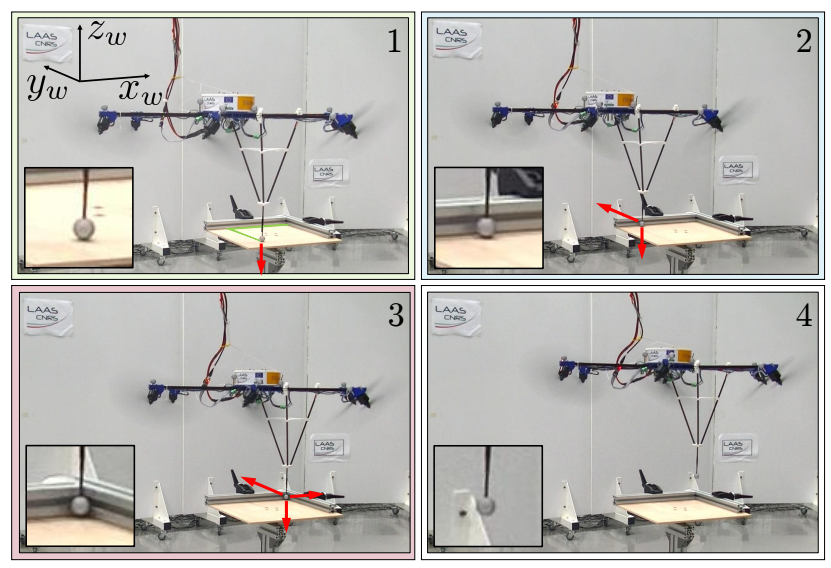

Figure 13. Snapshots of the multiple contacts experiment, contact forces are highlighted with red arrows. The background colors match the colors of the contact phases in Figure 14. 1) Single contact with surface. The Sliding trajectory is marked in green. 2.) Double Contact. 3.) Triple contact. 4.) Free flight phase
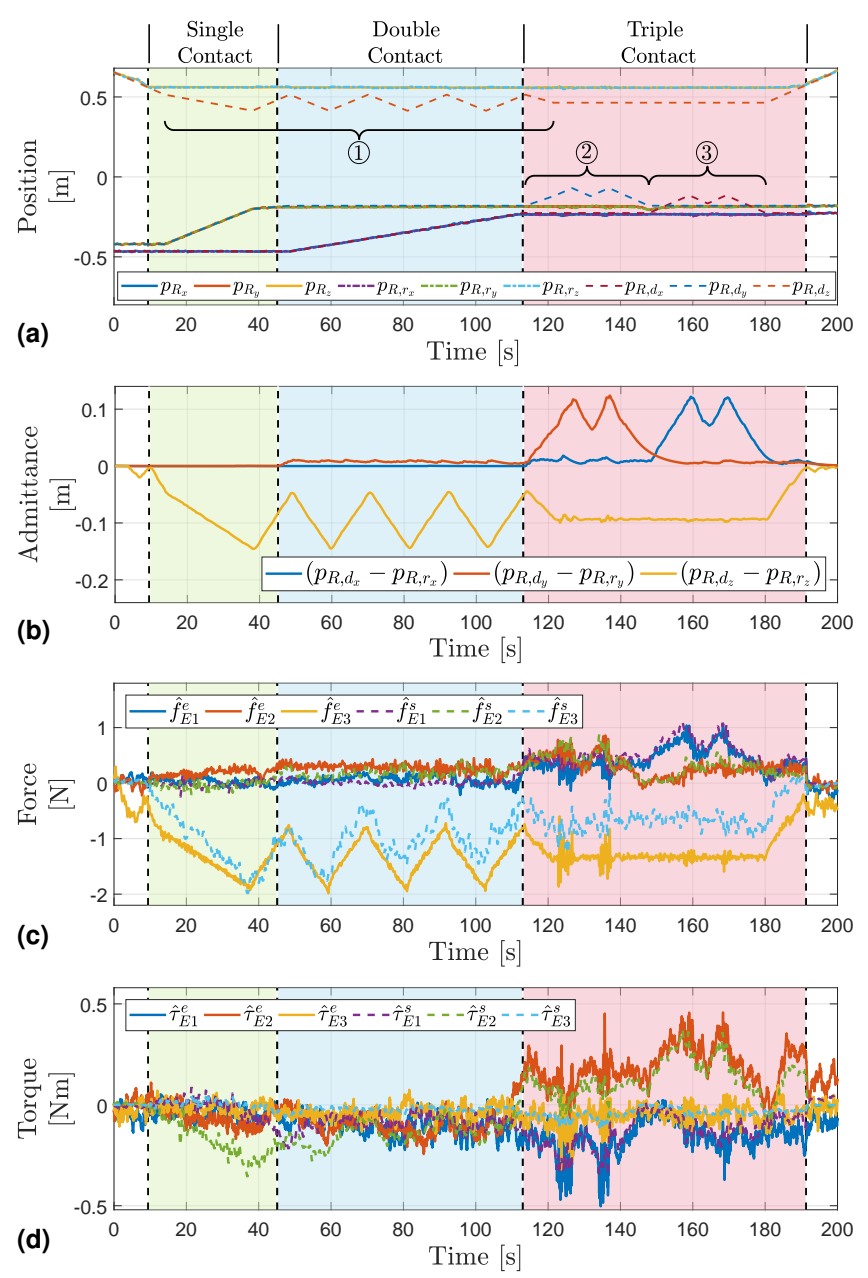

Figure 14. Multiple Contact Point task: a) Reference (dashed), desired (dot-dashed) and actual position (solid) b) Difference between desired trajectory $\mathbf{p}_{R, d}$ and the admittance filter output $\mathbf{p}_{R, r}$ due to the contact force. c) Estimated (solid) and measured (dashed) tool-tip contact forces - both low pass filtered. d) Estimated (solid) and measured (dashed) tool-tip contact torque - both low pass filtered. the admittance difference. In contrast to the first three experiments, aerodynamic effects between the aerial robot and the solid surface are observed, resulting in a mismatches between the estimated and measured forces and torques, see Figure 14c. While particularly for the horizontal force, the saw-tooth profile is clearly visible, the estimated force $\hat{f}_{E 3}^{e}$ and measured force $\hat{f}_{E 3}^{s}$ data drift apart, as the hexarotor flies over the platform. Interestingly the estimated force is larger than the measured value. We assume that the downwash of the propeller is reflected on the ground and lifts the surface, resulting in a wrong measured force $\hat{f}_{E 3}^{s}$. In order to complete the data presentation the estimated and measured torques are depicted in Figure 14d. While the trend of the estimated torques matches very well, we again see mismatches during phases where the propeller downwash particularly hits the plane far away from the center, where the force torque sensor is mounted (e.g., at $t=37 \mathrm{~s}$ ).

\section{Summary}

In this work, we tackled the issue of precise $6 \mathrm{D}$ aerial physical interaction with the environment. We utilized a novel fully actuated aerial platform, namely the Tilt-Hex allowing to independently control the linear and the angular acceleration. Hereby, the platform can instantaneously counteract any wrench during the contact with the environment. To achieve a bounded interaction wrench and a guaranteed stable behavior of the aerial platform a two-loop control framework has been developed. An outer loop admittance control scheme steers the system to a desired impedance behavior only during the presence of an interaction wrench by computing a suitable reference trajectory. The inner loop motion controller tracks the reference trajectory. An advanced set of experiments was performed to show the full capabilities of the platform.

In the future we seek for full autonomy of the system by replacing the motion capture system by full on-board state estimation. Furthermore we will work on differentiating contact forces on the tool-tip and disturbances on the platform (e.g., wind gusts).

\section{Acknowledgements}

This work has been funded by the European Union's Horizon 2020 research and innovation program under grant agreement No 644271 AEROARMS.

We thank Anthony Mallet (LAAS-CNRS) and Michelangelo Nigro (University of Basilicata) who collaborated to develop the software architecture (Antony Mallet) and to conduct some of the experiments (Michelangelo Nigro).

\section{References}

Antonelli G, Cataldi E, Muscio G, Trujillo M, Rodriguez Y, Pierri F, Caccavale F, Viguria A, Chiaverini S and Ollero A (2016) Impedance control of an aerial-manipulator: Preliminary results. In: 2016 IEEE/RSJ Int. Conf. on Intelligent Robots and Systems. Daejeon, South Korea, pp. 3848-3853.

Augugliaro F, Lupashin S, Hamer M, Male C, Hehn M, Mueller MW, Willmann JS, Gramazio F, Kohler M and D'Andrea R (2014) The flight assembled architecture installation: 
Cooperative construction with flying machines. IEEE Control Systems Magazine 34(4): 46-64.

Baizid K, Giglio G, Pierri F, Trujillo M, Antonelli G, Caccavale F, Viguria A, Chiaverini S and Ollero A (2016) Behavioral control of unmanned aerial vehicle manipulator systems. Autonomous Robots 35(8): 1-18.

Brescianini D and D'Andrea R (2016) Design, modeling and control of an omni-directional aerial vehicle. In: 2016 IEEE Int. Conf. on Robotics and Automation. Stockholm, Sweden, pp. 3261-3266.

De Luca A and Mattone R (2005) Sensorless robot collision detection and hybrid force/motion control. In: 2005 IEEE Int. Conf. on Robotics and Automation. Barcelona, Spain, pp. 9991004.

Faessler M, Franchi A and Scaramuzza D (2018) Differential flatness of quadrotor dynamics subject to rotor drag for accurate tracking of high-speed trajectories. IEEE Robot. Autom. Lett 3(2): 620-626.

Fink J, Michael N, Kim S and Kumar V (2011) Planning and control for cooperative manipulation and transportation with aerial robots. The International Journal of Robotics Research 30(3): 324-334.

Franchi A, Carli R, Bicego D and Ryll M (2018) Full-pose tracking control for aerial robotic systems with laterally bounded input force. IEEE Transactions on Robotics 34(2): 534-541.

Franchi A and Mallet A (2017) Adaptive closed-loop speed control of BLDC motors with applications to multi-rotor aerial vehicles. In: 2017 IEEE Int. Conf. on Robotics and Automation. Singapore, pp. 5203-5208.

Fumagalli M, Naldi R, Macchelli A, Carloni R, Stramigioli S and Marconi L (2012) Modeling and control of a flying robot for contact inspection. In: 2012 IEEE/RSJ Int. Conf. on Intelligent Robots and Systems. Vilamoura, Portugal, pp. 3532-3537.

Gioioso G, Franchi A, Salvietti G, Scheggi S and Prattichizzo D (2014a) The Flying Hand: a formation of UAVs for cooperative aerial tele-manipulation. In: 2014 IEEE Int. Conf. on Robotics and Automation. Hong Kong, China, pp. 4335-4341.

Gioioso G, Ryll M, Prattichizzo D, Bülthoff HH and Franchi A (2014b) Turning a near-hovering controlled quadrotor into a 3D force effector. In: 2014 IEEE Int. Conf. on Robotics and Automation. Hong Kong, China, pp. 6278-6284.

Kai JM, Allibert G, Hua MD and Hamel T (2017) Nonlinear feedback control of quadrotors exploiting first-order drag effects. IFAC-PapersOnLine 50(1): 8189-8195.

Khalil HK (2002) Nonlinear systems, volume 3. Prentice hall Upper Saddle River, NJ.

Kim S, Choi S and Kim HJ (2013) Aerial manipulation using a quadrotor with a two DOF robotic arm. In: 2013 IEEE/RSJ International Conference on Intelligent Robots and Systems. pp. 4990-4995. DOI:10.1109/IROS.2013.6697077.

Kim S, Seo H and Kim HJ (2015) Operating an unknown drawer using an aerial manipulator. In: Robotics and Automation (ICRA), 2015 IEEE International Conference on. IEEE, pp. 5503-5508.

Lee T, Leoky M and McClamroch NH (2010) Geometric tracking control of a quadrotor UAV on SE(3). In: 49th IEEE Conf. on Decision and Control. Atlanta, GA, pp. 5420-5425.

Long Y and Cappelleri DJ (2013) Omnicopter: A Novel Overactuated Micro Aerial Vehicle. Heidelberg: Springer International Publishing, pp. 215-226.
Michieletto G, Ryll M and Franchi A (2017) Control of statically hoverable multi-rotor aerial vehicles and application to rotorfailure robustness for hexarotors. In: 2017 IEEE Int. Conf. on Robotics and Automation. Singapore, pp. 2747-2752.

Muscio G, Pierri F, Trujillo MA, Cataldi E, Antonelli G, Caccavale F, Viguria A, Chiaverini S and Ollero A (2017) Coordinated control of aerial robotic manipulators: Theory and experiments. IEEE Transactions on Control Systems Technology PP(99): 18. DOI:10.1109/TCST.2017.2716905.

Muscio G, Pierri F, Trujillo MA, Cataldi E, Giglio G, Antonelli G, Caccavale F, Viguria A, Chiaverini S and Ollero A (2016) Experiments on coordinated motion of aerial robotic manipulators. In: 2016 IEEE Int. Conf. on Robotics and Automation. Stockholm, Sweden, pp. 1224-1229.

Nguyen H and Lee D (2013) Hybrid force/motion control and internal dynamics of quadrotors for tool operation. In: 2013 IEEE/RSJ Int. Conf. on Intelligent Robots and Systems. Tokyo, Japan, pp. 3458-3464.

Park S, J Her J, Kim J and Lee D (2016) Design, modeling and control of omni-directional aerial robot. In: 2016 IEEE/RSJ Int. Conf. on Intelligent Robots and Systems. Daejeon, South Korea, pp. 1570-1575.

Rajappa S, Bülthoff H and Stegagno P (2017) Design and implementation of a novel architecture for physical humanUAV interaction. The International Journal of Robotics Research : 0278364917708038.

Rajappa S, Ryll M, Bülthoff HH and Franchi A (2015) Modeling, control and design optimization for a fully-actuated hexarotor aerial vehicle with tilted propellers. In: 2015 IEEE Int. Conf. on Robotics and Automation. Seattle, WA, pp. 4006-4013.

Ryll M, Bicego D and Franchi A (2016) Modeling and control of FAST-Hex: A fully-actuated by synchronized-tilting hexarotor. In: 2016 IEEE/RSJ International Conference on Intelligent Robots and Systems (IROS). pp. 1689-1694. DOI:10.1109/ IROS.2016.7759271.

Ryll M, Bülthoff HH and Robuffo Giordano P (2015) A novel overactuated quadrotor unmanned aerial vehicle: modeling, control, and experimental validation. IEEE Trans. on Control Systems Technology 23(2): 540-556.

Ryll M, Muscio G, Pierri F, Cataldi E, Antonelli G, Caccavale F and Franchi A (2017) 6D physical interaction with a fully actuated aerial robot. In: 2017 IEEE Int. Conf. on Robotics and Automation. Singapore, pp. 5190-5195.

Siciliano B, Sciavicco L, Villani L and Oriolo G (2009) Robotics: Modelling, Planning and Control. Springer. ISBN 978-184628-641-4.

Tagliabue A, Kamel M, Verling S, Siegwart R and Nieto J (2016) Collaborative transportation using MAVs via passive force control. In: 2017 IEEE Int. Conf. on Robotics and Automation. Singapore, pp. 5766-5773.

Tognon M, Gabellieri C, Pallottino L and Franchi A (2018) Aerial co-manipulation with cables: The role of internal force for equilibria, stability, and passivity. IEEE Robotics and Automation Letters, Special Issue on Aerial Manipulation 3(3): 2577 - 2583. DOI:10.1109/LRA.2018.2803811.

Tognon M, Yüksel B, Buondonno G and Franchi A (2017) Dynamic decentralized control for protocentric aerial manipulators. In: 2017 IEEE Int. Conf. on Robotics and Automation. Singapore, pp. 6375-6380. 
Tomic T and Haddadin S (2014) A unified framework for external wrench estimation, interaction control and collision reflexes for flying robots. In: 2014 IEEE/RSJ International Conference on Intelligent Robots and Systems. pp. 4197-4204.

Tomic T, Ott C and Haddadin S (2017) External wrench estimation, collision detection, and reflex reaction for flying robots. IEEE Transactions on Robotics PP(99): 1-17. DOI:10.1109/TRO. 2017.2750703.

Villani L and De Schutter J (2008) Springer Handbook of Robotics, chapter Force Control. Heidelberg, D: B. Siciliano, O. Khatib, (Eds.), Springer-Verlag.

Voyles R and Jiang G (2014) A nonparallel hexrotor UAV with faster response to disturbances for precision position keeping. In: 2014 IEEE Int. Symp. on Safety, Security and Rescue Robotics. West Lafayette, IN, pp. 1-5.

Yang H and Lee D (2014) Dynamics and control of quadrotor with robotic manipulator. In: 2014 IEEE International Conference on Robotics and Automation (ICRA). pp. 5544-5549. DOI: 10.1109/ICRA.2014.6907674.

Yüksel B, Secchi C, Bülthoff HH and Franchi A (2014a) A nonlinear force observer for quadrotors and application to physical interactive tasks. In: 2014 IEEE/ASME Int. Conf. on Advanced Intelligent Mechatronics. Besançon, France, pp. 433-440.

Yüksel B, Secchi C, Bülthoff HH and Franchi A (2014b) Reshaping the physical properties of a quadrotor through IDA-PBC and its application to aerial physical interaction. In: 2014 IEEE Int. Conf. on Robotics and Automation. Hong Kong, China, pp. 6258-6265. 